\title{
IDEMPOTENT RESIDUATED STRUCTURES: SOME CATEGORY EQUIVALENCES AND THEIR APPLICATIONS
}

\author{
N. GALATOS AND J. G. RAFTERY
}

\begin{abstract}
This paper concerns residuated lattice-ordered idempotent commutative monoids that are subdirect products of chains. An algebra of this kind is a generalized Sugihara monoid (GSM) if it is generated by the lower bounds of the monoid identity; it is a Sugihara monoid if it has a compatible involution $\neg$. Our main theorem establishes a category equivalence between GSMs and relative Stone algebras with a nucleus (i.e., a closure operator preserving the lattice operations). An analogous result is obtained for Sugihara monoids. Among other applications, it is shown that Sugihara monoids are strongly amalgamable, and that the relevance logic $\mathbf{R} \mathbf{M}^{\mathbf{t}}$ has the projective Beth definability property for deduction.
\end{abstract}

\section{INTRODUCTION}

The theory of residuated structures descends from three essentially independent sources - the algebra of binary relations, the study of ideal multiplication in rings, and the semantic analysis of non-classical logics (see 23] and its references). In the structures that concern us here, the key ingredients are a commutative monoid $\langle A ; \cdot, \mathbf{t}\rangle$, a lattice order $\leq$ of $A$, and a binary operation $\rightarrow$ such that the law of residuation

$$
a \cdot c \leq b \text { iff } c \leq a \rightarrow b
$$

holds for all elements $a, b, c \in A$. When interpreting some deductive systems at the intersection of relevance logic and many-valued logic, we encounter the following properties as well:

- idempotence: $a \cdot a=a$ for all elements $a$,

- semilinearity: the structure is a subdirect product of chains.

None of these demands implies

- integrality: the monoid identity $\mathbf{t}$ is the greatest element.

On the whole, varieties of integral residuated structures are better understood than their non-integral counterparts, so the discovery of a category equivalence between a non-integral and an integral class may increase our understanding of the

Received by the editors February 23, 2012 and, in revised form, January 2, 2013.

2010 Mathematics Subject Classification. Primary 03B47, 03G25, 06F05; Secondary 03G27, $08 \mathrm{C} 05,08 \mathrm{C} 15$.

Key words and phrases. Residuation, idempotent, semilinear, representable, nucleus, Sugihara monoid, relative Stone algebra, category equivalence, epimorphism, amalgamation, Beth definability, interpolation, R-mingle.

The work of the first author was supported in part by Simons Foundation grant 245806 .

The second author was supported in part by the National Research Foundation of South Africa (UID 85407). 
former. Amalgamation and epimorphism-surjectivity properties will be preserved, for instance, because they are purely categorical. As it happens, they reflect interpolation theorems and definability properties of the deductive systems modeled by the algebras (see Theorem 11.2). Moreover, a category equivalence between varieties induces an isomorphism between their subvariety lattices, along which categorical properties can still be transferred. Thus, it carries positive and negative results from one family of logics to another, yielding swift new discoveries in some cases and easier proofs in others.

This strategy was exploited in [26], where we proved that the variety OSM of odd Sugihara monoids and the variety RSA of relative Stone algebras are categorically equivalent. Both classes consist of idempotent semilinear algebras. A residuated structure of this kind belongs to RSA iff it is integral; it is a Sugihara monoid iff it possesses a compatible involution $\neg$. The odd Sugihara monoids are the ones in which $\neg \mathbf{t}=\mathbf{t}$. Using known categorical properties of relative Stone algebras, we were able to establish some new features of the uninorm-based logic IUML (see [42,45]), which is algebraized by the bounded odd Sugihara monoids.

For the sake of such applications, it is desirable to extend the category equivalence in [26] to a wider class of residuated structures than OSM. The equivalence functor from OSM to RSA simply constructs the negative cone of the non-integral algebra, which is based on the lower bounds of $\mathbf{t}$. If this natural construction is still to be used, then we should deal with non-integral residuated structures that are determined by their negative cones. Assuming semilinearity and idempotence, the algebras generated by their negative elements are exactly the ones in which $(a \rightarrow \mathbf{t}) \rightarrow \mathbf{t}=a$ whenever $a \geq \mathbf{t}$. This demand defines the variety GSM of generalized Sugihara monoids, which is therefore a natural boundary for our investigation.

The negative cone of a generalized Sugihara monoid is still a relative Stone algebra, but the negative cone construction is no longer a category equivalence. To restore equivalence in the context of GSM, we must eliminate some RSA-morphisms by adding structure to the relative Stone algebras. We prove that it suffices to add a single unary operation $\diamond$ (called a nucleus), with axioms $x \leq \diamond x=\diamond \diamond x$ and $\diamond x \wedge \diamond y=\diamond(x \wedge y)$, where $\wedge$ is the lattice meet. Thus, we arrive at a variety NRSA of nuclear relative Stone algebras, and our central result shows that GSM and NRSA are categorically equivalent (Theorem 8.7). Because of the hereditary character of equivalence, we derive integral reductions of other non-integral classes too.

This allows us to show, for instance, that Sugihara monoids are strongly amalgamable, whence they enjoy a strong form of epimorphism-surjectivity. On the logical front, we obtain the finite Beth definability property for deduction in all axiomatic extensions of the relevance logic $\mathbf{R} \mathbf{M}^{\mathbf{t}}$ (that is, $\mathbf{R}-$ mingle, formulated with Ackermann constants [2]). For $\mathbf{R M}^{\mathbf{t}}$ itself, we obtain the projective Beth property for deduction, and a new proof of deductive interpolation. Finally, we generalize the following result from [49]: in the negation-less fragment of $\mathbf{R} \mathbf{M}^{\mathbf{t}}$, every extension is an axiomatic extension.

\section{Residuated StRuCtures}

An algebra $\boldsymbol{A}=\langle A ; \cdot, \rightarrow, \wedge, \vee, \mathbf{t}\rangle$ of type $\langle 2,2,2,2,0\rangle$ is called a commutative residuated lattice (briefly, a $C R L$ ) if $\langle A ; \cdot, \mathbf{t}\rangle$ is a commutative monoid, $\langle A ; \wedge, \vee\rangle$ is a lattice, and for all $a, b, c \in A$,

$$
c \leq a \rightarrow b \text { iff } a \cdot c \leq b,
$$


where $\leq$ denotes the lattice order. In this case, $\boldsymbol{A}$ also satisfies

$$
\begin{aligned}
& x \cdot(x \rightarrow y) \leq y, \\
& x \leq y \Longleftrightarrow \mathbf{t} \leq x \rightarrow y, \\
& x \leq y \Longrightarrow\left\{\begin{array}{l}
x \cdot z \leq y \cdot z \text { and } \\
z \rightarrow x \leq z \rightarrow y \text { and } \\
y \rightarrow z \leq x \rightarrow z
\end{array}\right. \\
& \mathbf{t} \rightarrow x=x, \\
& (x \cdot y) \rightarrow z=y \rightarrow(x \rightarrow z)=x \rightarrow(y \rightarrow z) .
\end{aligned}
$$

The class of all CRLs is an arithmetical variety with the congruence extension property 1, 22. Every CRL $\boldsymbol{A}$ is $\mathbf{t}-r e g u l a r$, i.e., each congruence $\theta$ of $\boldsymbol{A}$ is determined by the equivalence class $\mathbf{t} / \theta:=\left\{a \in A: a \equiv_{\theta} \mathbf{t}\right\}$. This follows from the quasi-equations

$$
(x \leftrightarrow y) \wedge \mathbf{t}=\mathbf{t} \Longleftrightarrow x=y,
$$

which are valid in all CRLs, where $x \leftrightarrow y:=(x \rightarrow y) \wedge(y \rightarrow x)$. For additional background on CRLs; see [23, 29].

\section{Semilinearity AND idempotence}

A CRL $\boldsymbol{A}$ is said to be distributive if its lattice reduct is distributive; it is semilinear if it can be embedded into a direct product of totally ordered CRLs. In the latter case, $\boldsymbol{A}$ is actually a subdirect product of totally ordered CRLs (because total order persists in subalgebras) and, when verifying that $\boldsymbol{A}$ satisfies a quasiequation, we may assume without loss of generality that $\langle A ; \leq\rangle$ is a chain.

The semilinear CRLs can be characterized as the distributive ones that satisfy

$$
\mathbf{t} \leq(x \rightarrow y) \vee(y \rightarrow x),
$$

whence they form a variety [29]. In the literature, 'representable' is a common synonym for 'semilinear'.

Whereas every CRL satisfies the distribution laws

$$
\begin{aligned}
& x \cdot(y \vee z)=(x \cdot y) \vee(x \cdot z), \\
& x \rightarrow(y \wedge z)=(x \rightarrow y) \wedge(x \rightarrow z), \\
& (x \vee y) \rightarrow z=(x \rightarrow z) \wedge(y \rightarrow z),
\end{aligned}
$$

the semilinear ones also satisfy

$$
\begin{aligned}
& x \cdot(y \wedge z)=(x \cdot y) \wedge(x \cdot z), \\
& x \rightarrow(y \vee z)=(x \rightarrow y) \vee(x \rightarrow z), \\
& (x \wedge y) \rightarrow z=(x \rightarrow z) \vee(y \rightarrow z)
\end{aligned}
$$

The following lemma is easily verified (see [26] if necessary).

Lemma 3.1. Let $\boldsymbol{A}$ be a semilinear $C R L$-or more generally, a CRL satisfying (5). Then $\boldsymbol{A}$ satisfies $x=(x \wedge \mathbf{t}) \cdot(x \vee \mathbf{t})$.

We adopt the abbreviations

$$
x^{*}:=x \rightarrow \mathbf{t} \text { and }|x|:=x \rightarrow x .
$$


Every CRL satisfies

$$
x \leq x^{* *} \text { and } x^{* * *}=x^{*} \text { and } \mathbf{t} \leq|x| .
$$

If a CRL is idempotent (i.e., it satisfies $x \cdot x=x$ ), then it also satisfies

$$
\begin{aligned}
& x \leq|x|, \\
& x=|x| \Longleftrightarrow \mathbf{t} \leq x, \\
& x^{*}=|x| \Longleftrightarrow x \leq \mathbf{t}, \\
& x=x^{*} \Longleftrightarrow x=\mathbf{t} .
\end{aligned}
$$

A CRL $\boldsymbol{A}$ is finitely subdirectly irreducible iff its identity element $\mathbf{t}$ is joinirreducible in the lattice reduct $\langle A ; \wedge, \vee\rangle$; see [24. If the set $\{a \in A: a<\mathbf{t}\}$ has a greatest element, then $\boldsymbol{A}$ is subdirectly irreducible. The converse holds when $\boldsymbol{A}$ is idempotent - and somewhat more generally [48. Thus, by Jónsson's Lemma (see 34] or [12, Thm. IV.6.8]),

a semilinear idempotent CRL $\boldsymbol{A}$ is finitely subdirectly irreducible iff $\langle A ; \leq\rangle$ is a chain; it is subdirectly irreducible iff $\langle A ; \leq\rangle$ is a chain in which $\mathbf{t}$ is the cover of some element.

This remains true for CRL-expansions (i.e., CRLs, possibly enriched with extra operations), provided that $\boldsymbol{A}$ and its CRL-reduct have the same congruences.

The variety of semilinear idempotent CRLs is locally finite, i.e., every finitely generated member of this class is a finite algebra [54. A totally ordered idempotent CRL $\boldsymbol{A}$ is determined by its reduct $\left\langle A ;^{*}, \leq\right\rangle$; it is also determined by $\left\langle A ;\left.\right|_{-} \mid, \leq\right\rangle$. These claims follow from (9)-(11) and the next theorem.

Theorem 3.2 ([54]). Let $\boldsymbol{A}$ be a totally ordered idempotent CRL. Then

(i) A satisfies

$$
x \cdot y=\left\{\begin{array}{ll}
x & \text { if }|y|<|x| \\
y & \text { if }|x|<|y| \\
x \wedge y & \text { if }|x|=|y|
\end{array} \quad \text { and } \quad x \rightarrow y= \begin{cases}x^{*} \vee y & \text { if } x \leq y \\
x^{*} \wedge y & \text { if } x>y\end{cases}\right.
$$

(ii) Let $\mathbf{t} \leq a \in A$ and define

$$
Y_{a}=\left\{c \in A: c^{*}=a\right\}, \text { i.e., } Y_{a}=\{c \in A: c \leq \mathbf{t} \text { and }|c|=a\} .
$$

Then $Y_{a}$ is an interval of $\langle A ; \leq\rangle$. Also, $Y_{a} \neq \emptyset$ iff $a^{* *}=a$, in which case $a^{*}$ is the greatest element of $Y_{a}$.

An element $a$ of a CRL will be called negative if $a \leq \mathbf{t}$, and positive if $\mathbf{t} \leq a$. Given a totally ordered idempotent CRL $\boldsymbol{A}$, the non-empty sets of the form $Y_{a}(a$ positive) clearly partition the negative elements. Thus, by Theorem $3.2, \boldsymbol{A}$ will be generated as an algebra by its negative elements iff $a^{* *}=a$ for all positive $a \in A$. This motivates the next definition.

Definition 3.3. The variety GSM of generalized Sugihara monoids consists of the semilinear idempotent CRLs that satisfy

$$
(x \vee \mathbf{t})^{* *}=x \vee \mathbf{t},
$$

or equivalently, $\mathbf{t} \leq x \Longrightarrow x^{* *}=x$. (The reason for this name will become clear in Section 5]) The algebras in GSM are therefore just the subdirect products of totally ordered idempotent CRLs $\boldsymbol{A}$ in which $Y_{a} \neq \emptyset$ for all positive $a \in A$. 


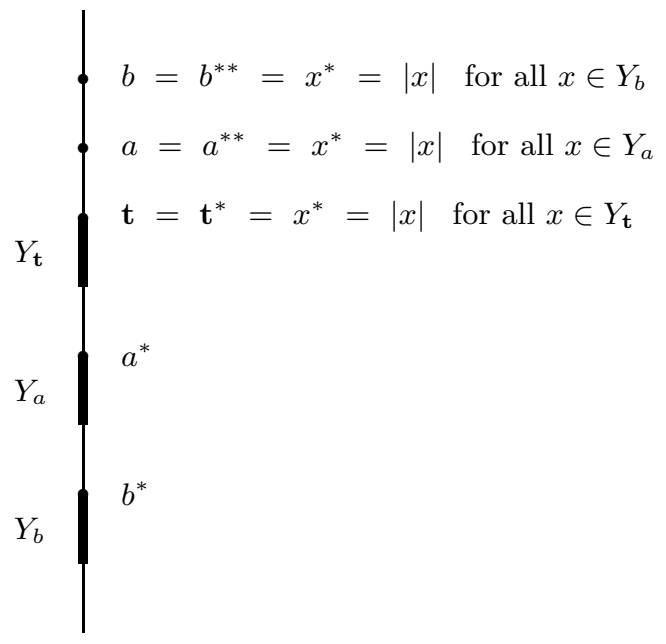

FiguRE 1. A totally ordered member of GSM, illustrating Theorem 3.2(iii)

Lemma 3.4. Every generalized Sugihara monoid satisfies

$$
x=(x \wedge \mathbf{t}) \cdot\left(x^{*} \wedge \mathbf{t}\right)^{*} .
$$

Proof. By (4), every CRL satisfies $(x \vee \mathbf{t})^{*}=x^{*} \wedge \mathbf{t}^{*}=x^{*} \wedge \mathbf{t}$, so GSM satisfies $x \vee \mathbf{t}=(x \vee \mathbf{t})^{* *}=\left(x^{*} \wedge \mathbf{t}\right)^{*}$, and the result follows from Lemma 3.1

Corollary 3.5. A semilinear idempotent CRL is a generalized Sugihara monoid iff it is generated by its negative elements.

Proof. The forward implication follows from Lemma 3.4. By semilinearity, the converse need only be checked in the totally ordered case, as surjective homomorphisms always map generating sets onto generating sets. Thus, the remarks before Definition 3.3 complete the proof.

\section{Relative Stone algebras}

An integral CRL is one whose identity element $\mathbf{t}$ is its greatest element. In these algebras, we always have $a \leq b \rightarrow a$, while $a \leq b$ iff $a \rightarrow b=\mathbf{t}$; in particular, $a \rightarrow \mathbf{t}=\mathbf{t}$ and (10) simplifies to

$$
x \leftrightarrow y=\mathbf{t} \Longleftrightarrow x=y .
$$

A Brouwerian algebra is an integral idempotent CRL, i.e., a CRL in which $a \cdot b=a \wedge b$ for all elements $a, b$. Clearly, these algebras have the contraction property $x \rightarrow(x \rightarrow y)=x \rightarrow y$.

Every totally ordered Brouwerian algebra satisfies

$$
\begin{aligned}
& x \rightarrow y=\left\{\begin{array}{ll}
\mathbf{t} & \text { if } x \leq y ; \\
y & \text { if } x>y
\end{array}\right. \text { and } \\
& (x \rightarrow y) \rightarrow x=\left\{\begin{array}{lll}
x & \text { if } x \leq y \\
\mathbf{t} & \text { if } x>y
\end{array}\right.
\end{aligned}
$$


The variety RSA of relative Stone algebras consists of the semilinear Brouwerian algebras. Thus, RSA is the class of all integral members of GSM and it is very well understood. If $\boldsymbol{A} \in \mathrm{RSA}$ is totally ordered, then, in the notation of Theorem 3.2(iii), we have $Y_{\mathbf{t}}=A$.

Lemma 4.1. For any elements $a, b$ of a relative Stone algebra, the following conditions are equivalent:

(i) $a \rightarrow b=b$ and $b \rightarrow a=a$;

(ii) $a \vee b=\mathbf{t}$.

In a totally ordered relative Stone algebra, these conditions are equivalent to

(iii) $a=\mathbf{t}$ or $b=\mathbf{t}$.

Proof. Clearly, the lemma's second assertion follows from its first, and the first need only be verified in the totally ordered case. The result is therefore an easy consequence of (14).

Lemma 4.2. RSA satisfies $((x \rightarrow y) \rightarrow x) \vee(x \rightarrow y)=\mathbf{t}$.

Proof. Again, an examination of the totally ordered algebras suffices, and the result follows readily from (14) and (15).

\section{InVOlution AND Sugihara MONOIDS}

An involutive $C R L$ is the expansion of a CRL $\boldsymbol{A}$ by a basic unary operation $\neg$ such that $\neg \neg a=a$ and $a \rightarrow \neg b=b \rightarrow \neg a$ for all $a, b \in A$. In this case, the De Morgan laws for $\neg, \wedge, \vee$ hold as well. Involutive CRLs still have the congruence extension property, because they are termwise equivalent to CRLs with a distinguished element $\mathbf{f}$ such that $(a \rightarrow \mathbf{f}) \rightarrow \mathbf{f}=a$ for all elements $a$. (Define $\mathbf{f}=\neg \mathbf{t}$ in one direction, and $\neg a=a \rightarrow \mathbf{f}$ in the other.) In an idempotent involutive CRL, we always have $\mathbf{f} \leq \mathbf{t}$.

An involutive CRL $\boldsymbol{A}$ is said to be odd if $\neg \mathbf{t}=\mathbf{t}$, i.e., if $a^{* *}=a$ for all $a \in A$. In this case, $\boldsymbol{A}$ is termwise equivalent to its CRL-reduct, as $\neg a=a^{*}$ for all $a \in A$.

\section{Definition 5.1.}

(i) The variety SM of Sugihara monoids consists of the idempotent distributive involutive CRLs.

(ii) The class PSM of positive Sugihara monoids consists of the CRLs $\boldsymbol{A}$ that can be extended to Sugihara monoids (i.e., $\boldsymbol{A}$ is a subalgebra of the $\cdot, \rightarrow, \wedge, \vee, \mathbf{t}$ reduct of some Sugihara monoid).

(iii) OSM denotes the variety of odd Sugihara monoids.

J. M. Dunn, in his contributions to [2, showed that Sugihara monoids are semilinear. In fact, SM is the smallest variety containing the unique Sugihara monoid

$$
\boldsymbol{Z}-\{0\}=\langle\{a: 0 \neq a \in \mathbb{Z}\} ; \cdot, \rightarrow, \wedge, \vee,-, 1\rangle
$$

on the set of all non-zero integers such that the lattice order is the usual total order, the involution - is the usual additive inversion, and the term function of $|x|:=x \rightarrow x$ is the natural absolute value function. In this algebra,

$$
a \cdot b=\left\{\begin{array}{l}
\text { the element of }\{a, b\} \text { with the greater absolute value, if }|a| \neq|b| \\
a \wedge b \text { if }|a|=|b|,
\end{array}\right.
$$


and the residual operation $\rightarrow$ is given by

$$
a \rightarrow b= \begin{cases}(-a) \vee b & \text { if } a \leq b ; \\ (-a) \wedge b & \text { if } a \leq \leq b\end{cases}
$$

(cf. Theorem 3.2). Note that $\mathbf{t}$ is 1 and $\mathbf{f}$ is -1 in $\boldsymbol{Z}-\{0\}$, so $(-1)^{*}=1$. Although $\boldsymbol{Z}-\{0\}$ is not odd, it has only one element $a$ for which $a^{* *} \neq a$, namely $a=-1$. In particular, the identity (12) is valid in $\boldsymbol{Z}-\{0\}$, and hence in SM. It follows that

$$
\mathrm{OSM}^{+} \subseteq \mathrm{PSM} \subseteq \mathrm{GSM}
$$

where $\mathrm{OSM}^{+}$is the variety of CRL-reducts of odd Sugihara monoids. Both inclusions are strict. It is shown in [49] that PSM is itself a variety.

In the Sugihara monoid $\boldsymbol{Z}=\langle\mathbb{Z} ; \cdot, \rightarrow, \wedge, \vee,-, 0\rangle$ on the set of all integers, the operations are defined like those of $\boldsymbol{Z}-\{0\}$, except that 0 takes over from 1 as the identity element for $\cdot$. Both $\mathbf{t}$ and $\mathbf{f}$ are 0 in $\boldsymbol{Z}$, so $\boldsymbol{Z} \in$ OSM.

It follows from Dunn's results in 2 that OSM is the smallest quasivariety containing $\boldsymbol{Z}$, and that SM is the smallest quasivariety containing both $\boldsymbol{Z}-\{0\}$ and $\boldsymbol{Z}$. Observe that $\boldsymbol{Z}$ is a homomorphic image of $\boldsymbol{Z}-\{0\}$. The kernel of the homomorphism identifies -1 with 1 ; it identifies no other pair of distinct elements. We cannot embed $\boldsymbol{Z}$ into $\boldsymbol{Z}-\{0\}$, owing to the involution. Nevertheless, the CRLreduct of $\boldsymbol{Z}$ is isomorphic to the subalgebra on $\mathbb{Z}-\{-1,0\}$ of the CRL-reduct of $\boldsymbol{Z}-\{0\}$. Consequently, PSM is the smallest quasivariety containing the CRL-reduct of $\boldsymbol{Z}-\{0\}$.

\section{Categorical equivalence}

Recall that two categories $C$ and $D$ are said to be equivalent if there are functors $F: \mathrm{C} \longrightarrow \mathrm{D}$ and $G: \mathrm{D} \longrightarrow \mathrm{C}$ such that $F \circ G$ and $G \circ F$ are naturally isomorphic to the identity functors on $\mathrm{D}$ and $\mathrm{C}$, respectively. In the concrete category associated with a class of similar algebras, the objects are the members of the class, and the morphisms are all the algebraic homomorphisms between pairs of objects. The set of homomorphisms from $\boldsymbol{A}$ into $\boldsymbol{B}$ is denoted, as usual, by $\operatorname{Hom}(\boldsymbol{A}, \boldsymbol{B})$. Two isomorphically-closed classes of similar algebras, $\mathrm{C}$ and $\mathrm{D}$, are said to be categorically equivalent if the corresponding concrete categories are equivalent. For this, it is sufficient (and necessary) that some functor $F: C \longrightarrow D$ should have the following properties:

(i) for each $\boldsymbol{U} \in \mathrm{D}$, there exists $\boldsymbol{A} \in \mathrm{C}$ with $F(\boldsymbol{A}) \cong \boldsymbol{U}$, and

(ii) the map $h \mapsto F(h)$ from $\operatorname{Hom}(\boldsymbol{A}, \boldsymbol{B})$ to $\operatorname{Hom}(F(\boldsymbol{A}), F(\boldsymbol{B}))$ is bijective, for all $\boldsymbol{A}, \boldsymbol{B} \in \mathrm{C}$.

In this case, $F$ and some functor $G$ from $\mathrm{D}$ to $C$ witness the equivalence of these concrete categories. We call $G$ a reverse functor for $F$, and vice versa. Note that $\mathrm{C}$ and $\mathrm{D}$ are not assumed to have the same algebraic similarity type.

In [26], we proved that OSM is categorically equivalent to RSA. As categorical properties often reflect metalogical features of deductive systems, it would be desirable to extend this correspondence beyond OSM - for instance to the variety SM of all Sugihara monoids, or at least to PSM. These classes model the well-known system $\mathbf{R M}^{\mathbf{t}}$ from relevance logic [2,18, and its negation-less fragment. 
The equivalence functor from OSM to RSA is the 'negative cone' functor. In general, the negative cone of a CRL $\boldsymbol{A}=\langle A ; \cdot, \rightarrow, \wedge, \vee, \mathbf{t}\rangle$ is the integral CRL

$$
\boldsymbol{A}^{-}=\left\langle A^{-} ; \cdot^{-}, \rightarrow^{-}, \wedge^{-}, \vee^{-}, \mathbf{t}\right\rangle
$$

on the set $A^{-}:=\{a \in A: a \leq \mathbf{t}\}$, where $\cdot^{-}, \wedge^{-}, \vee^{-}$are just the respective restrictions of $\cdot, \wedge, \vee$ to $A^{-} \times A^{-}$, and the residual $\rightarrow^{-}$is given by

$$
a \rightarrow^{-} b=(a \rightarrow b) \wedge \mathbf{t} \text { for all } a, b \in A^{-} .
$$

(If $\boldsymbol{A}$ is an involutive CRL, then $\boldsymbol{A}^{-}$denotes the negative cone of the CRL-reduct of $\boldsymbol{A}$.)

The negative cone of a positive Sugihara monoid is still a relative Stone algebra, but PSM and RSA are not categorically equivalent, as their subvariety lattices are not isomorphic (see Theorem 9.2 below). To restore equivalence, we need to eliminate some RSA-morphisms by adding structure to the relative Stone algebras. We shall prove that it is sufficient to add a single unary operation, which is a nucleus in the sense of the next section, having certain properties. But it makes sense to try to extend the domain of the equivalence from PSM to the widest possible variety of CRLs, relaxing the properties of the nucleus accordingly. If a negative cone construction is still to be used, then the non-integral algebras should be determined by their negative elements. Since a semilinear idempotent CRL is generated by its negative elements iff it is a generalized Sugihara monoid, the variety GSM is a natural boundary for this investigation. We shall show that GSM is categorically equivalent to the variety of all relative Stone algebras with an arbitrary nucleus (Theorem 8.7).

Once established, the desired equivalence can be restricted to subvarieties of GSM, including PSM. The algebras in GSM (and PSM) need not have an involution, however, and every involutive algebra in GSM is in fact a Sugihara monoid. Therefore, we shall also establish a category equivalence between Sugihara monoids and another variety of enriched relative Stone algebras. For that purpose, we shall add a special constant (as well as a nucleus) to the type of RSA; see Theorem 10.5 .

\section{NUCLEI}

A nucleus of a CRL $\boldsymbol{A}$ is a function $N: A \longrightarrow A$ such that, for all $a, b \in A$,

$$
\begin{aligned}
& a \leq N a=N N a, \\
& \text { if } a \leq b, \text { then } N a \leq N b, \\
& N a \cdot N b \leq N(a \cdot b) .
\end{aligned}
$$

Nuclei are used extensively in the theory of residuated structures, particularly in connection with embedding problems; see 23. A nuclear $C R L$ is the expansion of a CRL $\boldsymbol{A}$ by a nucleus $N$. In this case, for all $a, b \in A$, we have

$$
a \rightarrow b \leq N a \rightarrow N b,
$$

because $N a \cdot(a \rightarrow b) \leq N a \cdot N(a \rightarrow b) \leq N(a \cdot(a \rightarrow b)) \leq N b$. Consequently, $N$ is compatible with every congruence $\theta$ of $\boldsymbol{A}$. Indeed, since $\boldsymbol{A} / \theta$ is again a CRL, it follows from (11) that, for all $a, b \in A$,

$$
a \equiv_{\theta} b \text { iff }(a \leftrightarrow b) \wedge \mathbf{t} \equiv_{\theta} \mathbf{t} .
$$


So, if $a \equiv_{\theta} b$, then

$$
\begin{aligned}
& (N a \leftrightarrow N b) \wedge \mathbf{t} \equiv_{\theta}(N a \leftrightarrow N b) \wedge(a \leftrightarrow b) \wedge \mathbf{t}[\text { by (20) }] \\
& =(a \leftrightarrow b) \wedge \mathbf{t}[\text { by (19) }] \equiv_{\theta} \mathbf{t},
\end{aligned}
$$

whence $N a \equiv_{\theta} N b$. In other words:

Theorem 7.1. A nuclear $C R L \boldsymbol{B}$ and its $C R L$-reduct $\boldsymbol{A}$ always have the same congruences. In particular, $\boldsymbol{B}$ is [finitely] subdirectly irreducible iff $\boldsymbol{A}$ is.

Notation. For a class C of CRLs, we use NC to denote the class of all nuclear CRLs $\langle\boldsymbol{A}, N\rangle$ such that $\boldsymbol{A} \in \mathrm{C}$.

The inequalities in (16)-(18) can be rendered as equations involving $\wedge$, and the definition of a nucleus can be made purely equational, because (17) can be replaced by $N(a \wedge b) \leq N b$. This, with Theorem 7.1 establishes the following facts.

Corollary 7.2. If $\mathrm{V}$ is a variety of CRLs, then NV is also a variety, and NV is arithmetical, congruence extensible and $\mathbf{t}-$ regular.

In this case, if $\boldsymbol{B} \in \mathrm{NV}$ and the CRL-reduct of $\boldsymbol{B}$ is semilinear, then $\boldsymbol{B}$ is a subdirect product of totally ordered members of NV.

Now suppose $\boldsymbol{A}$ is a Brouwerian algebra and $N$ a nucleus of $\boldsymbol{A}$. It is easy to see that $N(a \wedge b)=N a \wedge N b$ for all $a, b \in A$, but $N$ need not preserve joins. When $\boldsymbol{A}$ is a relative Stone algebra, however, the law $N(a \vee b)=N a \vee N b$ follows from the semilinearity of $\boldsymbol{A}$ and the isotonicity of $N$. So, in this case, $N$ is an idempotent endomorphism (i.e., a retraction) of the lattice reduct of $\boldsymbol{A}$. In summary: a unary operation $N$ on a relative Stone algebra $\boldsymbol{A}$ is a nucleus iff it is a retraction of the lattice reduct of $\boldsymbol{A}$ and $a \leq N a$ for all $a \in A$.

In the variety NRSA of nuclear relative Stone algebras, we tend to denote the nuclear operation as $\diamond$, rather than $N$, because it is a closure operator that preserves joins.

Example 7.3. For every CRL $\boldsymbol{A}$, if $a \in A^{-}$, then $a^{* *} \in A^{-}$. When $\boldsymbol{A}$ is semilinear and idempotent, then $\boldsymbol{A}^{-} \in \mathrm{RSA}$, and a nucleus of $\boldsymbol{A}^{-}$is defined by $\nabla a=a^{* *}$ (i.e., $\diamond a=\left(a \rightarrow^{\boldsymbol{A}} \mathbf{t}\right) \rightarrow^{\boldsymbol{A}} \mathbf{t}$ for all $\left.a \in A^{-}\right)$. This nucleus need not be a term function of $\boldsymbol{A}^{-}$. We use $\boldsymbol{A}_{\diamond}^{-}$to denote the resulting algebra $\left\langle\boldsymbol{A}^{-}, \diamond\right\rangle \in$ NRSA, which we call the nuclear negative cone of $\boldsymbol{A}$.

Notation. If $\boldsymbol{A}$ is a Sugihara monoid, then $\boldsymbol{A}_{\diamond}^{-}$stands for the nuclear negative cone of the CRL-reduct of $\boldsymbol{A}$. If $\mathrm{C}$ is a class of [involutive] CRLs, then $\mathrm{C}^{-}$shall abbreviate $\left\{\boldsymbol{A}^{-}: \boldsymbol{A} \in \mathrm{C}\right\}$ and, where appropriate, $\mathrm{C}_{\diamond}^{-}:=\left\{\boldsymbol{A}_{\diamond}^{-}: \boldsymbol{A} \in \mathrm{C}\right\}$.

Our immediate aim is to prove that GSM and NRSA are categorically equivalent. Example 7.3 gives us a way to associate a nuclear relative Stone algebra $\boldsymbol{A}_{\diamond}^{-}$with a given generalized Sugihara monoid $\boldsymbol{A}$. The construction becomes a functor from GSM to NRSA if we also restrict GSM-morphisms to the negative cones of their domains. We call this the nuclear negative cone functor. It is much less obvious how to construct a reverse functor from NRSA to GSM. That will be done in the next section. 


\section{A FUNCTOR FROM NRSA TO GSM}

Let $\boldsymbol{A}=\langle A ; \wedge, \rightarrow, \wedge, \vee, \diamond, \mathbf{t}\rangle$ be a nuclear relative Stone algebra, where, as usual, $\leq$ denotes the lattice order of $\boldsymbol{A}$. We define

$$
S(\boldsymbol{A})=\left\{\left\langle a, a^{\prime}\right\rangle \in A \times A: a \vee a^{\prime}=\mathbf{t} \text { and } \diamond a^{\prime}=a^{\prime}\right\} .
$$

Thus, by Lemma 4.1 .

$$
S(\boldsymbol{A})=\left\{\left\langle a, a^{\prime}\right\rangle \in A \times A: a \rightarrow a^{\prime}=a^{\prime}=\diamond a^{\prime} \text { and } a^{\prime} \rightarrow a=a\right\} .
$$

Let $\left\langle a, a^{\prime}\right\rangle,\left\langle b, b^{\prime}\right\rangle \in S(\boldsymbol{A})$. We define

$$
\begin{aligned}
\left\langle a, a^{\prime}\right\rangle \wedge\left\langle b, b^{\prime}\right\rangle & =\left\langle a \wedge b, a^{\prime} \vee b^{\prime}\right\rangle, \\
\left\langle a, a^{\prime}\right\rangle \vee\left\langle b, b^{\prime}\right\rangle & =\left\langle a \vee b, a^{\prime} \wedge b^{\prime}\right\rangle, \\
\left\langle a, a^{\prime}\right\rangle \cdot\left\langle b, b^{\prime}\right\rangle & =\left\langle\left(\left(a \rightarrow b^{\prime}\right) \wedge\left(b \rightarrow a^{\prime}\right)\right) \rightarrow(a \wedge b), \diamond\left(\left(a \rightarrow b^{\prime}\right) \wedge\left(b \rightarrow a^{\prime}\right)\right)\right\rangle, \\
\left\langle a, a^{\prime}\right\rangle \rightarrow\left\langle b, b^{\prime}\right\rangle & =\left\langle(a \rightarrow b) \wedge\left(b^{\prime} \rightarrow a^{\prime}\right), \diamond\left(\left((a \rightarrow b) \wedge\left(b^{\prime} \rightarrow a^{\prime}\right)\right) \rightarrow\left(a \wedge b^{\prime}\right)\right)\right\rangle .
\end{aligned}
$$

The genesis of these definitions will be discussed in Section 14.

To see that $S(\boldsymbol{A})$ is closed under $\wedge$, observe that

$$
(a \wedge b) \vee\left(a^{\prime} \vee b^{\prime}\right)=\left(a \vee a^{\prime} \vee b^{\prime}\right) \wedge\left(b \vee a^{\prime} \vee b^{\prime}\right) \geq\left(a \vee a^{\prime}\right) \wedge\left(b \vee b^{\prime}\right)=\mathbf{t}
$$

and $\diamond\left(a^{\prime} \vee b^{\prime}\right)=\diamond a^{\prime} \vee \diamond b^{\prime}=a^{\prime} \vee b^{\prime}$, because $\left\langle a, a^{\prime}\right\rangle,\left\langle b, b^{\prime}\right\rangle \in S(\boldsymbol{A})$. Similarly, $S(\boldsymbol{A})$ is closed under $\vee$.

Regarding closure under $\cdot$, let $m=\left(a \rightarrow b^{\prime}\right) \wedge\left(b \rightarrow a^{\prime}\right)$. Since $\diamond \diamond m=\diamond m$, we need only show that $(m \rightarrow(a \wedge b)) \vee \diamond m=\mathbf{t}$. By Corollary 7.2, $\boldsymbol{A}$ is a subdirect product of totally ordered nuclear Brouwerian algebras, so it suffices to prove the equality under the assumption that $\boldsymbol{A}$ is totally ordered. Then, by Lemma 4.1, $a$ or $a^{\prime}$ is $\mathbf{t}$, and $b$ or $b^{\prime}$ is t t, because $\left\langle a, a^{\prime}\right\rangle,\left\langle b, b^{\prime}\right\rangle \in S(\boldsymbol{A})$. If $a=b=\mathbf{t}$, then $m \rightarrow(a \wedge b)=\mathbf{t}$, and if $a^{\prime}=b^{\prime}=\mathbf{t}$, then $m=\mathbf{t}$, so the result holds in these two cases. If $a=b^{\prime}=\mathbf{t}$, then the equation to be proved is $\left(\left(b \rightarrow a^{\prime}\right) \rightarrow b\right) \vee \diamond\left(b \rightarrow a^{\prime}\right)=\mathbf{t}$, and, because NRSA satisfies $x \leq \diamond x$, it suffices to prove $\left(\left(b \rightarrow a^{\prime}\right) \rightarrow b\right) \vee\left(b \rightarrow a^{\prime}\right)=\mathbf{t}$. This follows from Lemma 4.2 Finally, if $a^{\prime}=b=\mathbf{t}$, then the result follows from the previous case, by symmetry.

Thus, $S(\boldsymbol{A})$ is closed under $\cdot$. The proof that $S(\boldsymbol{A})$ is closed under $\rightarrow$ is very similar. Clearly, $\langle\mathbf{t}, \mathbf{t}\rangle \in S(\boldsymbol{A})$, so we may consider the algebra

$$
\boldsymbol{S}(\boldsymbol{A})=\langle S(\boldsymbol{A}) ; \cdot, \rightarrow, \wedge, \vee,\langle\mathbf{t}, \mathbf{t}\rangle\rangle .
$$

Theorem 8.1. If $\boldsymbol{A}$ is a nuclear relative Stone algebra, then $\boldsymbol{S}(\boldsymbol{A})$ is a generalized Sugihara monoid.

Proof. Because $\langle A ; \wedge, \vee\rangle$ is a distributive lattice, so is $\langle S(\boldsymbol{A}) ; \wedge, \vee\rangle$. The lattice order of $\boldsymbol{S}(\boldsymbol{A})$ is just

$$
\left\langle a, a^{\prime}\right\rangle \leq\left\langle b, b^{\prime}\right\rangle \text { iff }\left(a \leq b \text { and } b^{\prime} \leq a^{\prime}\right) .
$$

Note that $\cdot$ is commutative on $S(\boldsymbol{A})$, by symmetry. Also, $\cdot$ idempotent with identity $\langle\mathbf{t}, \mathbf{t}\rangle:$ from $a \rightarrow a^{\prime}=a^{\prime}=\diamond a^{\prime}$ and $a^{\prime} \rightarrow a=a$, we infer

$$
\left\langle a, a^{\prime}\right\rangle \cdot\left\langle a, a^{\prime}\right\rangle=\left\langle\left(a \rightarrow a^{\prime}\right) \rightarrow a, \diamond\left(a \rightarrow a^{\prime}\right)\right\rangle=\left\langle a^{\prime} \rightarrow a, \diamond a^{\prime}\right\rangle=\left\langle a, a^{\prime}\right\rangle,
$$

and similarly, $\left\langle a, a^{\prime}\right\rangle \cdot\langle\mathbf{t}, \mathbf{t}\rangle=\left\langle a, a^{\prime}\right\rangle$.

For associativity of $\cdot$, let $u=\left\langle a, a^{\prime}\right\rangle, v=\left\langle b, b^{\prime}\right\rangle$ and $w=\left\langle c, c^{\prime}\right\rangle$ be elements of $S(\boldsymbol{A})$, so $a \vee a^{\prime}=b \vee b^{\prime}=c \vee c^{\prime}=\mathbf{t}$ and $\nabla a^{\prime}=a^{\prime}$ and $\diamond b^{\prime}=b^{\prime}$ and $\diamond c^{\prime}=c^{\prime}$. Let

$$
\langle p, q\rangle=u \cdot(v \cdot w) \text { and }\langle r, s\rangle=(u \cdot v) \cdot w \text {. }
$$


Each of $p, q, r, s$ has the form $f^{\boldsymbol{A}}\left(a, a^{\prime}, b, b^{\prime}, c, c^{\prime}\right)$ for some term $f$ in the language of CRLs. So, by the subdirect decomposition, it suffices to prove that $\langle p, q\rangle=\langle r, s\rangle$ under the assumption that $\boldsymbol{A}$ is totally ordered. This gives rise to eight cases, which reduce to the following four independent cases, because $\cdot$ is commutative:

$$
\begin{aligned}
& \left\langle\mathbf{t}, a^{\prime}\right\rangle \cdot\left(\left\langle\mathbf{t}, b^{\prime}\right\rangle \cdot\left\langle\mathbf{t}, c^{\prime}\right\rangle\right)=\left(\left\langle\mathbf{t}, a^{\prime}\right\rangle \cdot\left\langle\mathbf{t}, b^{\prime}\right\rangle\right) \cdot\left\langle\mathbf{t}, c^{\prime}\right\rangle, \\
& \left\langle\mathbf{t}, a^{\prime}\right\rangle \cdot\left(\left\langle\mathbf{t}, b^{\prime}\right\rangle \cdot\langle c, \mathbf{t}\rangle\right)=\left(\left\langle\mathbf{t}, a^{\prime}\right\rangle \cdot\left\langle\mathbf{t}, b^{\prime}\right\rangle\right) \cdot\langle c, \mathbf{t}\rangle, \\
& \left\langle\mathbf{t}, a^{\prime}\right\rangle \cdot(\langle b, \mathbf{t}\rangle \cdot\langle c, \mathbf{t}\rangle)=\left(\left\langle\mathbf{t}, a^{\prime}\right\rangle \cdot\langle b, \mathbf{t}\rangle\right) \cdot\langle c, \mathbf{t}\rangle, \\
& \langle a, \mathbf{t}\rangle \cdot(\langle b, \mathbf{t}\rangle \cdot\langle c, \mathbf{t}\rangle)=(\langle a, \mathbf{t}\rangle \cdot\langle b, \mathbf{t}\rangle) \cdot\langle c, \mathbf{t}\rangle .
\end{aligned}
$$

In the first of these equations, both sides simplify to $\left\langle\mathbf{t}, a^{\prime} \wedge b^{\prime} \wedge c^{\prime}\right\rangle$; in the last, both sides become $\langle a \wedge b \wedge c, \mathbf{t}\rangle$. The second and third equations boil down to

$$
\begin{aligned}
& \left\langle k \rightarrow \diamond\left(\left(c \rightarrow b^{\prime}\right) \rightarrow c\right), \diamond k\right\rangle=\left\langle\left(c \rightarrow\left(b^{\prime} \wedge a^{\prime}\right)\right) \rightarrow c, \diamond\left(c \rightarrow\left(b^{\prime} \wedge a^{\prime}\right)\right)\right\rangle \text { and } \\
& \langle\ell \rightarrow(b \wedge c), \diamond \ell\rangle=\left\langle\left(c \rightarrow \diamond\left(b \rightarrow a^{\prime}\right)\right) \rightarrow\left(\left(\left(b \rightarrow a^{\prime}\right) \rightarrow b\right) \wedge c\right), \diamond\left(c \rightarrow \diamond\left(b \rightarrow a^{\prime}\right)\right)\right\rangle,
\end{aligned}
$$

respectively, where

$$
k:=\diamond\left(c \rightarrow b^{\prime}\right) \wedge\left(\left(\left(c \rightarrow b^{\prime}\right) \rightarrow c\right) \rightarrow a^{\prime}\right) \text { and } \ell:=(b \wedge c) \rightarrow a^{\prime} .
$$

The reader should separate the cases $c \leq b^{\prime}$ and $c>b^{\prime}$ when checking the second equation. In the third, separate $b \leq a^{\prime}$ from $b>a^{\prime}$. Properties (14) and (15) are useful in both verifications.

Next, we establish the residuation axiom

$$
w \leq u \rightarrow v \text { iff } u \cdot w \leq v
$$

where $u, v, w$ are as above. This amounts to showing that, of the four conditions below, the first two are jointly equivalent to the last two:

$$
\begin{aligned}
& c \leq(a \rightarrow b) \wedge\left(b^{\prime} \rightarrow a^{\prime}\right) \\
& \diamond\left(\left((a \rightarrow b) \wedge\left(b^{\prime} \rightarrow a^{\prime}\right)\right) \rightarrow\left(a \wedge b^{\prime}\right)\right) \leq c^{\prime} \\
& \left(\left(a \rightarrow c^{\prime}\right) \wedge\left(c \rightarrow a^{\prime}\right)\right) \rightarrow(a \wedge c) \leq b \\
& b^{\prime} \leq \diamond\left(\left(a \rightarrow c^{\prime}\right) \wedge\left(c \rightarrow a^{\prime}\right)\right) .
\end{aligned}
$$

Again, we may assume without loss of generality that $\boldsymbol{A}$ is totally ordered.

Suppose (23) and (24) hold, and note that (23) yields

$$
a \wedge c \leq b \text { and } b^{\prime} \wedge c \leq a^{\prime} .
$$

We shall prove (26) first. Since $\boldsymbol{A}$ satisfies $x \leq \nabla x$, it suffices to show that $b^{\prime} \leq$ $a \rightarrow c^{\prime}$ and $b^{\prime} \leq c \rightarrow a^{\prime}$. The latter is an immediate consequence of the second inequality in (27). For the former, set

$$
d:=\left((a \rightarrow b) \wedge\left(b^{\prime} \rightarrow a^{\prime}\right)\right) \rightarrow\left(a \wedge b^{\prime}\right) .
$$

Then $a \wedge b^{\prime} \leq d$ (as $\boldsymbol{A}$ is integral) $\leq \diamond d \leq c^{\prime}$ (by (24) $)$. So, $b^{\prime} \leq a \rightarrow c^{\prime}$, completing the proof of (26). Note that (25) will be true if $b=\mathbf{t}$, so assume $b \neq \mathbf{t}$. Then $b^{\prime}=\mathbf{t}$, because $b \vee b^{\prime}=\mathbf{t}$ and $\boldsymbol{A}$ is totally ordered. Now $c \leq a^{\prime}$, by (27), i.e., $c \rightarrow a^{\prime}=\mathbf{t}$. If $c^{\prime}=\mathbf{t}$, then

$$
\left(\left(a \rightarrow c^{\prime}\right) \wedge\left(c \rightarrow a^{\prime}\right)\right) \rightarrow(a \wedge c)=\mathbf{t} \rightarrow(a \wedge c)=a \wedge c \leq b,
$$

by (27), i.e., (25) holds. We may therefore assume that $c^{\prime} \neq \mathbf{t}$, whence $c=\mathbf{t}$, and so $a^{\prime}=\mathbf{t}\left(\right.$ as $\left.c \leq a^{\prime}\right)$. Now, using integrality and (24) again, we have

$$
a \leq(a \rightarrow b) \rightarrow a=d \leq \diamond d \leq c^{\prime},
$$


i.e., $a \rightarrow c^{\prime}=\mathbf{t}$. This allows us to repeat the calculation in (28), thus completing the proof of (25).

Conversely, assume (25) and (26). Then

$$
a \wedge c \leq\left(\left(a \rightarrow c^{\prime}\right) \wedge\left(c \rightarrow a^{\prime}\right)\right) \rightarrow(a \wedge c) \leq b,
$$

by (25), so $c \leq a \rightarrow b$. Also,

$$
\begin{aligned}
b^{\prime} \wedge c & \leq c \wedge \diamond\left(\left(a \rightarrow c^{\prime}\right) \wedge\left(c \rightarrow a^{\prime}\right)\right) \quad(\text { by (26) }) \\
& \leq \diamond c \wedge \diamond\left(c \rightarrow a^{\prime}\right)=\diamond\left(c \wedge\left(c \rightarrow a^{\prime}\right)\right) \leq \diamond a^{\prime}=a^{\prime},
\end{aligned}
$$

so $c \leq b^{\prime} \rightarrow a^{\prime}$, whence (23) holds. With a view to proving (24), let

$$
g:=\left((a \rightarrow b) \wedge\left(b^{\prime} \rightarrow a^{\prime}\right)\right) \rightarrow\left(a \wedge b^{\prime}\right) .
$$

We must show that $\nabla g \leq c^{\prime}$. This will be true if $c^{\prime}=\mathbf{t}$, so assume that $c^{\prime} \neq \mathbf{t}$, hence $c=\mathbf{t}$. We cannot have $a=b^{\prime}=\mathbf{t}$, as that would lead, via (26) , to the contradiction $\mathbf{t}=\diamond\left(c^{\prime} \wedge a^{\prime}\right) \leq \nabla c^{\prime}=c^{\prime}$. If $b=a^{\prime}=\mathbf{t}$, then (26) yields $b^{\prime} \leq \diamond\left(a \rightarrow c^{\prime}\right)$, whence

$$
\diamond g=\diamond\left(a \wedge b^{\prime}\right) \leq \diamond\left(a \wedge \diamond\left(a \rightarrow c^{\prime}\right)\right)=\diamond\left(a \wedge\left(a \rightarrow c^{\prime}\right)\right) \leq \diamond c^{\prime}=c^{\prime},
$$

as required. If $a=b=\mathbf{t}$, then (26) gives $b^{\prime} \leq \diamond\left(c^{\prime} \wedge a^{\prime}\right)=\diamond c^{\prime} \wedge \diamond a^{\prime}=c^{\prime} \wedge a^{\prime}$, so

$$
\begin{aligned}
\diamond g & =\diamond\left(\left(b^{\prime} \rightarrow a^{\prime}\right) \rightarrow b^{\prime}\right) \leq \diamond\left(\left(\left(c^{\prime} \wedge a^{\prime}\right) \rightarrow a^{\prime}\right) \rightarrow\left(c^{\prime} \wedge a^{\prime}\right)\right) \\
& =\diamond\left(\mathbf{t} \rightarrow\left(c^{\prime} \wedge a^{\prime}\right)\right)=\diamond\left(c^{\prime} \wedge a^{\prime}\right) \leq \diamond c^{\prime}=c^{\prime} .
\end{aligned}
$$

Finally, suppose $a^{\prime}=b^{\prime}=\mathbf{t}$. If $a>c^{\prime}$, then (26) and (14) give $\mathbf{t}=\diamond c^{\prime}=c^{\prime}$, whence $\diamond g \leq c^{\prime}$. So, assume $a \leq c^{\prime}$. Then (25) entails that $a \leq b$, whence

$$
\diamond g=\diamond a \leq \diamond c^{\prime}=c^{\prime},
$$

as required. We have now shown that $\boldsymbol{S}(\boldsymbol{A})$ is a (distributive) CRL.

Because $\boldsymbol{S}(\boldsymbol{A})$ is distributive, it will be semilinear if

$$
\langle\mathbf{t}, \mathbf{t}\rangle \leq\left(\left\langle a, a^{\prime}\right\rangle \rightarrow\left\langle b, b^{\prime}\right\rangle\right) \vee\left(\left\langle b, b^{\prime}\right\rangle \rightarrow\left\langle a, a^{\prime}\right\rangle\right)
$$

for all $\left\langle a, a^{\prime}\right\rangle,\left\langle b, b^{\prime}\right\rangle \in S(\boldsymbol{A})$. This translates, via (21), into two inequalities, one for each co-ordinate. Since $\mathbf{t}$ is the greatest element of $\boldsymbol{A}$, the claim for the second co-ordinate is trivial. In the first co-ordinate, we need to show that

$$
\mathbf{t}=\left((a \rightarrow b) \wedge\left(b^{\prime} \rightarrow a^{\prime}\right)\right) \vee\left((b \rightarrow a) \wedge\left(a^{\prime} \rightarrow b^{\prime}\right)\right) .
$$

Once again, we may assume that $\boldsymbol{A}$ is totally ordered. Now (29) will hold when $a=b=t$ and when $a^{\prime}=b^{\prime}=\mathbf{t}$, because $\boldsymbol{A}$ satisfies $(x \rightarrow y) \vee(y \rightarrow x)=\mathbf{t}$. In the remaining cases, viz. $a=b^{\prime}=\mathbf{t}$ or $a^{\prime}=b=\mathbf{t}$, (29) is true as well, because it asserts that $\mathbf{t}=\mathbf{t} \vee h$ for a certain $h \in A$. Thus, $\boldsymbol{S}(\boldsymbol{A})$ is semilinear.

Observe that, for all $\left\langle a, a^{\prime}\right\rangle \in S(\boldsymbol{A})$, we have

$$
\left\langle a, a^{\prime}\right\rangle^{*}=\left\langle a^{\prime}, \diamond a\right\rangle \text {. }
$$

Indeed, $\left\langle a, a^{\prime}\right\rangle^{*}=\left\langle\mathbf{t} \wedge a^{\prime}, \diamond\left(\left(\mathbf{t} \wedge a^{\prime}\right) \rightarrow(a \wedge \mathbf{t})\right)\right\rangle=\left\langle a^{\prime}, \diamond\left(a^{\prime} \rightarrow a\right)\right\rangle=\left\langle a^{\prime}, \diamond a\right\rangle$.

To see that $\boldsymbol{S}(\boldsymbol{A}) \in \mathrm{GSM}$, suppose $\langle\mathbf{t}, \mathbf{t}\rangle \leq\left\langle a, a^{\prime}\right\rangle \in S(\boldsymbol{A})$, so $a=\mathbf{t}$, by (21). We must show that $\left\langle\mathbf{t}, a^{\prime}\right\rangle^{* *}=\left\langle\mathbf{t}, a^{\prime}\right\rangle$. By (130), $\left\langle\mathbf{t}, a^{\prime}\right\rangle^{*}=\left\langle a^{\prime}, \diamond \mathbf{t}\right\rangle=\left\langle a^{\prime}, \mathbf{t}\right\rangle$, so $\left\langle\mathbf{t}, a^{\prime}\right\rangle^{* *}=\left\langle a^{\prime}, \mathbf{t}\right\rangle^{*}=\left\langle\mathbf{t}, \diamond a^{\prime}\right\rangle=\left\langle\mathbf{t}, a^{\prime}\right\rangle$, as required.

The universe $S(\boldsymbol{A})^{-}$of the negative cone of $\boldsymbol{S}(\boldsymbol{A})$ is $\{\langle a, \mathbf{t}\rangle: a \in A\}$, by (21).

Theorem 8.2. If $\boldsymbol{A}$ is a nuclear relative Stone algebra, then $\boldsymbol{A} \cong \boldsymbol{S}(\boldsymbol{A})_{\diamond}^{-}$, the isomorphism being $a \mapsto\langle a, \mathbf{t}\rangle$. 
Proof. Obviously, $a \mapsto\langle a, \mathbf{t}\rangle$ is a bijection from $A$ to $S(\boldsymbol{A})^{-}$that preserves $\wedge, \vee$ and $\mathbf{t}$. It remains to note that if $a, b \in A$, then

$$
\begin{aligned}
& \langle a, \mathbf{t}\rangle \rightarrow^{-}\langle b, \mathbf{t}\rangle=\langle a \rightarrow b, \mathbf{t}\rangle, \text { and } \\
& \langle a, \mathbf{t}\rangle^{* *}=\langle\diamond a, \mathbf{t}\rangle .
\end{aligned}
$$

Indeed, $(\langle a, \mathbf{t}\rangle \rightarrow\langle b, \mathbf{t}\rangle) \wedge\langle\mathbf{t}, \mathbf{t}\rangle=\langle a \rightarrow b, \diamond((a \rightarrow b) \rightarrow a)\rangle \wedge\langle\mathbf{t}, \mathbf{t}\rangle=\langle a \rightarrow b, \mathbf{t}\rangle$, and, by (30), $\langle a, \mathbf{t}\rangle^{* *}=\langle\mathbf{t}, \diamond a\rangle^{*}=\langle\diamond a, \diamond \mathbf{t}\rangle=\langle\diamond a, \mathbf{t}\rangle$.

Lemma 8.3. Let $\boldsymbol{A}$ be a nuclear relative Stone algebra, with $\left\langle a, a^{\prime}\right\rangle \in S(\boldsymbol{A})$. Then

$$
\left\langle a, a^{\prime}\right\rangle=\langle a, \mathbf{t}\rangle \cdot\left\langle\mathbf{t}, a^{\prime}\right\rangle .
$$

Proof. This is a special case of Lemma 3.1, in view of Theorem 8.1. Alternatively, $a \rightarrow a^{\prime}=a^{\prime}=\diamond a^{\prime}$ and $a^{\prime} \rightarrow a=a$, by assumption, so

$$
\langle a, \mathbf{t}\rangle \cdot\left\langle\mathbf{t}, a^{\prime}\right\rangle=\left\langle\left(a \rightarrow a^{\prime}\right) \rightarrow a, \diamond\left(a \rightarrow a^{\prime}\right)\right\rangle=\left\langle a^{\prime} \rightarrow a, \diamond a^{\prime}\right\rangle=\left\langle a, a^{\prime}\right\rangle .
$$

Example 8.4. Suppose $\boldsymbol{A} \in$ NRSA is based on the chain $d<c<\mathbf{t}$. If $\diamond^{\boldsymbol{A}}$ is the identity function, then $\boldsymbol{S}(\boldsymbol{A})$ is based on the following chain $X$ :

$$
\langle d, \mathbf{t}\rangle<\langle c, \mathbf{t}\rangle<\langle\mathbf{t}, \mathbf{t}\rangle<\langle\mathbf{t}, c\rangle<\langle\mathbf{t}, d\rangle .
$$

In this case, $\boldsymbol{S}(\boldsymbol{A})$ is isomorphic to the CRL-subreduct of $\boldsymbol{Z}$ on

$$
-2<-1<0<1<2,
$$

or equivalently to the CRL-subreduct of $\boldsymbol{Z}-\{0\}$ on $-3<-2<1<2<3$. 1 On the other hand, if $\diamond d=d$ and $\nabla c=\mathbf{t}$, then $\boldsymbol{S}(\boldsymbol{A})$ is based on $X-\{\langle\mathbf{t}, c\rangle\}$ and it matches $-2<-1<1<2$ in the CRL-reduct of $\boldsymbol{Z}-\{0\}$. If $\nabla d=\nabla c=\mathbf{t}$, then $\boldsymbol{S}(\boldsymbol{A}) \cong \boldsymbol{A}$. Finally, if $\nabla d=c$, then $\boldsymbol{A}$ is the free 1-generated algebra in NRSA, while $\boldsymbol{S}(\boldsymbol{A})$ is based on $X-\{\langle\mathbf{t}, d\rangle\}$ and is generated by $\langle d, \mathbf{t}\rangle$. In this case, $\boldsymbol{S}(\boldsymbol{A})$ is isomorphic to the (unique) algebra in GSM freely generated by a single element $a$ subject to the relation $a \wedge \mathbf{t}=a$. These facts will be needed in Corollary 14.4.

Theorem 8.5. Let $\boldsymbol{A}$ be a generalized Sugihara monoid. Then $\boldsymbol{A} \cong \boldsymbol{S}\left(\boldsymbol{A}_{\diamond}^{-}\right)$. The isomorphism $h$ is given by $a \mapsto\left\langle a \wedge \mathbf{t}, a^{*} \wedge \mathbf{t}\right\rangle$.

Proof. Note first that $h(a) \in S\left(\boldsymbol{A}_{\diamond}^{-}\right)$for all $a \in A$, because

$$
\begin{aligned}
& (a \wedge \mathbf{t}) \vee^{-}\left(a^{*} \wedge \mathbf{t}\right)=(a \wedge \mathbf{t}) \vee\left(a^{*} \wedge \mathbf{t}\right) \\
= & \left(a \vee a^{*}\right) \wedge \mathbf{t}=((\mathbf{t} \rightarrow a) \vee(a \rightarrow \mathbf{t})) \wedge \mathbf{t}=\mathbf{t},
\end{aligned}
$$

by the semilinearity of $\boldsymbol{A}$, while $\diamond\left(a^{*} \wedge \mathbf{t}\right)=\left(a^{*} \wedge \mathbf{t}\right)^{* *}=a^{* * *} \wedge \mathbf{t}^{* *}=a^{*} \wedge \mathbf{t}$, by (7) and (4).

It follows from Lemma 3.4 that $h$ is one-to-one. To see that it is onto, let $\left\langle a, a^{\prime}\right\rangle \in S\left(\boldsymbol{A}_{\diamond}^{-}\right)$, so $\mathbf{t} \geq a, a^{\prime} \in A$ and $a \vee a^{\prime}=a \vee^{-} a^{\prime}=\mathbf{t}$ and $\diamond a^{\prime}=a^{\prime}$. Let $b=\left(a \rightarrow a^{\prime}\right) \rightarrow a$. We claim that $h(b)=\left\langle a, a^{\prime}\right\rangle$, i.e., that

$$
\left\langle\left(\left(a \rightarrow a^{\prime}\right) \rightarrow a\right) \wedge \mathbf{t},\left(\left(a \rightarrow a^{\prime}\right) \rightarrow a\right)^{*} \wedge \mathbf{t}\right\rangle=\left\langle a, a^{\prime}\right\rangle .
$$

Because $\boldsymbol{A}$ is semilinear, it suffices to prove this under the assumption that $\boldsymbol{A}$ is totally ordered, whence $a$ or $a^{\prime}$ is $\mathbf{t}$. If $a=\mathbf{t}$, then, since $a^{\prime} \leq \mathbf{t}$, we have $b=a^{*} \geq \mathbf{t}$ and $b^{*}=a^{* *}=\diamond a^{\prime}=a^{\prime}$, hence

$$
h(b)=\left\langle a^{* *} \wedge \mathbf{t}, a^{\prime} \wedge \mathbf{t}\right\rangle=\left\langle\mathbf{t}, a^{\prime}\right\rangle=\left\langle a, a^{\prime}\right\rangle .
$$

\footnotetext{
${ }^{1}$ A subreduct of an algebra $\boldsymbol{A}$ is a subalgebra of the indicated reduct of $\boldsymbol{A}$.
} 
On the other hand, if $a^{\prime}=\mathbf{t}>a$, then $a^{*} \geq \mathbf{t}$, whence $a^{*}>a$, so by Theorem 3.2(1), $b=a^{*} \rightarrow a=a^{* *} \wedge a=a$. In this case,

$$
h(b)=\left\langle a \wedge \mathbf{t}, a^{*} \wedge \mathbf{t}\right\rangle=\langle a, \mathbf{t}\rangle=\left\langle a, a^{\prime}\right\rangle,
$$

so $h$ is indeed onto.

Clearly, $h(\mathbf{t})=\langle\mathbf{t}, \mathbf{t}\rangle$. To see that $h$ preserves $\vee$ and $\wedge$, use the fact that semilinear CRLs satisfy $(x \vee y)^{*}=x^{*} \wedge y^{*}$ and $(x \wedge y)^{*}=x^{*} \vee y^{*}$ (by (4) and (7)), as well as the distributive laws for $\wedge, \vee$.

To show that $h$ preserves $\cdot$, let $a, b \in A$. As $a \wedge \mathbf{t}, b \wedge \mathbf{t} \leq \mathbf{t}$, we have $\mathbf{t} \leq$ $(a \wedge \mathbf{t})^{*},(b \wedge \mathbf{t})^{*}$. The desired result $h(a) \cdot h(b)=h(a \cdot b)$ boils down to

$$
\left\langle u \rightarrow^{-}(a \wedge b \wedge \mathbf{t}), \diamond u\right\rangle=\left\langle(a \cdot b) \wedge \mathbf{t},(a \cdot b)^{*} \wedge \mathbf{t}\right\rangle,
$$

where

$$
\begin{aligned}
u & :=\left((a \wedge \mathbf{t}) \rightarrow^{-}\left(b^{*} \wedge \mathbf{t}\right)\right) \wedge\left((b \wedge \mathbf{t}) \rightarrow^{-}\left(a^{*} \wedge \mathbf{t}\right)\right) \\
& =\left((a \wedge \mathbf{t}) \rightarrow\left(b^{*} \wedge \mathbf{t}\right)\right) \wedge\left((b \wedge \mathbf{t}) \rightarrow\left(a^{*} \wedge \mathbf{t}\right)\right) \wedge \mathbf{t} \\
& =\left((a \wedge \mathbf{t}) \rightarrow b^{*}\right) \wedge(a \wedge \mathbf{t})^{*} \wedge\left((b \wedge \mathbf{t}) \rightarrow a^{*}\right) \wedge(b \wedge \mathbf{t})^{*} \wedge \mathbf{t} \quad(\text { by (3) }) \\
& =\left((a \wedge \mathbf{t}) \rightarrow b^{*}\right) \wedge\left((b \wedge \mathbf{t}) \rightarrow a^{*}\right) \wedge \mathbf{t} \\
& =\left(\left(a \rightarrow b^{*}\right) \vee b^{*}\right) \wedge\left(\left(b \rightarrow a^{*}\right) \vee a^{*}\right) \wedge \mathbf{t} \quad(\text { by (7) }) .
\end{aligned}
$$

Again, we may assume that $\boldsymbol{A}$ is totally ordered.

Suppose first that $a \leq b^{*}$. This means that $a \cdot b \leq \mathbf{t}$, whence $b \leq a^{*}$. Thus, $\mathbf{t} \leq a \rightarrow b^{*}, b \rightarrow a^{*}$, and so $u=\mathbf{t}$. Recall that $a \cdot b \in\{a \wedge b, a \vee b\}$, by Theorem 3.2(i). But $a \cdot b=a \cdot a \cdot b \leq a \cdot \mathbf{t}=a$ and $a \cdot b=a \cdot b \cdot b \leq \mathbf{t} \cdot b=b$, so $a \cdot b \leq a \wedge b$, hence $a \cdot b=a \wedge b$. Now

$$
\begin{aligned}
& u \rightarrow^{-}(a \wedge b \wedge \mathbf{t})=(\mathbf{t} \rightarrow(a \cdot b)) \wedge \mathbf{t}=(a \cdot b) \wedge \mathbf{t}, \\
& \text { and }(a \cdot b)^{*} \wedge \mathbf{t}=((a \cdot b) \rightarrow \mathbf{t}) \wedge \mathbf{t}=\mathbf{t}=\diamond u,
\end{aligned}
$$

because $a \cdot b \leq \mathbf{t}=u$. In other words, (34) holds when $a \leq b^{*}$.

We may therefore assume that $a \not \leq b^{*}$, i.e., $a \cdot b \not \mathbf{t}$, i.e., $b \not \leq a^{*}$, so $b^{*}<a$ and $\mathbf{t}<a \cdot b$ and $a^{*}<b$. Dualizing the argument in the previous paragraph, we obtain $a \cdot b=a \vee b$. Also, $a \rightarrow b^{*}=a^{*} \wedge b^{*}$ and $b \rightarrow a^{*}=b^{*} \wedge a^{*}$, by Theorem 3.2(1). So, $u=\left(\left(a^{*} \wedge b^{*}\right) \vee b^{*}\right) \wedge\left(\left(b^{*} \wedge a^{*}\right) \vee a^{*}\right) \wedge \mathbf{t}=b^{*} \wedge a^{*} \wedge \mathbf{t}$. It follows that

$$
\diamond u=u^{* *}=u=(a \vee b)^{*} \wedge \mathbf{t}=(a \cdot b)^{*} \wedge \mathbf{t} .
$$

Also, $u \leq a \wedge b \wedge \mathbf{t}$, because $b^{*}<a$ and $a^{*}<b$. So,

$$
u \rightarrow^{-}(a \wedge b \wedge \mathbf{t})=(u \rightarrow(a \wedge b \wedge \mathbf{t})) \wedge \mathbf{t}=\mathbf{t}=(a \cdot b) \wedge \mathbf{t},
$$

i.e., (34) holds. Thus, $h$ preserves $\cdot$.

We have shown that $h$ is an isomorphism between the lattice-ordered monoid reducts of $\boldsymbol{A}$ and $\boldsymbol{S}\left(\boldsymbol{A}_{\diamond}^{-}\right)$. In each of these reducts, we already know that $x \rightarrow y$ is always the largest $z$ for which $x \cdot z \leq y$, so $\rightarrow$ is first order definable in terms of $\cdot \wedge$. Therefore, $h(a \rightarrow b)=h(a) \rightarrow h(b)$ for all $a, b \in A$, whence $h$ is an isomorphism of generalized Sugihara monoids.

Theorem 8.6. Let $\boldsymbol{A}$ and $\boldsymbol{B}$ be nuclear relative Stone algebras.

(i) If $h: \boldsymbol{A} \longrightarrow \boldsymbol{B}$ is a homomorphism, then $S(h):\left\langle a, a^{\prime}\right\rangle \mapsto\left\langle h(a), h\left(a^{\prime}\right)\right\rangle$ is a homomorphism from $\boldsymbol{S}(\boldsymbol{A})$ into $\boldsymbol{S}(\boldsymbol{B})$.

(ii) The map $h \mapsto S(h)$ is a bijection from $\operatorname{Hom}(\boldsymbol{A}, \boldsymbol{B})$ to $\operatorname{Hom}(\boldsymbol{S}(\boldsymbol{A}), \boldsymbol{S}(\boldsymbol{B}))$. 
Proof. (1) follows straightforwardly from the definitions of the operations.

(iii) If $a \in A$, then $\langle a, \mathbf{t}\rangle \in S(\boldsymbol{A})$. From this it follows easily that the function $h \mapsto S(h)$ is injective on $\operatorname{Hom}(\boldsymbol{A}, \boldsymbol{B})$.

For surjectivity, consider $g \in \operatorname{Hom}(\boldsymbol{S}(\boldsymbol{A}), \boldsymbol{S}(\boldsymbol{B}))$. If $\mathbf{t}^{\boldsymbol{S}(\boldsymbol{A})} \geq w \in S(\boldsymbol{A})$, then $g(w) \leq g\left(\mathbf{t}^{\boldsymbol{S}(\boldsymbol{A})}\right)=\mathbf{t}^{\boldsymbol{S}(\boldsymbol{B})}$, so there is a function $\tilde{g}: A \longrightarrow B$ such that

$$
\langle\tilde{g}(a), \mathbf{t}\rangle=g(\langle a, \mathbf{t}\rangle) \text { for all } a \in A .
$$

We claim that $\tilde{g} \in \operatorname{Hom}(\boldsymbol{A}, \boldsymbol{B})$. This follows from the fact that $g$ is a homomorphism. For example, let $a, a^{\prime} \in A$. Then

$$
\begin{aligned}
& \left.\left\langle\tilde{g}\left(a \rightarrow a^{\prime}\right), \mathbf{t}\right\rangle=g\left(\left\langle a \rightarrow a^{\prime}, \mathbf{t}\right\rangle\right)=g\left(\langle a, \mathbf{t}\rangle \rightarrow^{-}\left\langle a^{\prime}, \mathbf{t}\right\rangle\right) \quad \text { (by (31) }\right) \\
& =g(\langle a, \mathbf{t}\rangle) \rightarrow^{-} g\left(\left\langle a^{\prime}, \mathbf{t}\right\rangle\right)=\langle\tilde{g}(a), \mathbf{t}\rangle \rightarrow^{-}\left\langle\tilde{g}\left(a^{\prime}\right), \mathbf{t}\right\rangle \\
& \left.=\left\langle\tilde{g}(a) \rightarrow \tilde{g}\left(a^{\prime}\right), \mathbf{t}\right\rangle \quad \text { (by (31) }\right),
\end{aligned}
$$

so $\tilde{g}\left(a \rightarrow a^{\prime}\right)=\tilde{g}(a) \rightarrow \tilde{g}\left(a^{\prime}\right)$, while

$$
\begin{aligned}
& \langle\tilde{g}(\diamond a), \mathbf{t}\rangle=g(\langle\nabla a, \mathbf{t}\rangle)=g\left(\langle a, \mathbf{t}\rangle^{* *}\right) \quad(\text { by }(\text { (32) }) \\
& =g(\langle a, \mathbf{t}\rangle)^{* *}=\langle\tilde{g}(a), \mathbf{t}\rangle^{* *}=\langle\diamond \tilde{g}(a), \mathbf{t}\rangle \quad(\text { by (32) }),
\end{aligned}
$$

whence $\tilde{g}(\diamond a)=\diamond \tilde{g}(a)$.

Moreover, if $\left\langle a, a^{\prime}\right\rangle \in S(\boldsymbol{A})$, then $\diamond a^{\prime}=a^{\prime}$, hence $\nabla \tilde{g}\left(a^{\prime}\right)=\tilde{g}\left(a^{\prime}\right)$, and so

$$
\left\langle a^{\prime}, \mathbf{t}\right\rangle^{*}=\left\langle\mathbf{t}, a^{\prime}\right\rangle \text { and }\left\langle\tilde{g}\left(a^{\prime}\right), \mathbf{t}\right\rangle^{*}=\left\langle\mathbf{t}, \tilde{g}\left(a^{\prime}\right)\right\rangle,
$$

by (30). In this case, by (33) and (35),

$$
\begin{aligned}
& g\left(\left\langle a, a^{\prime}\right\rangle\right)=g\left(\langle a, \mathbf{t}\rangle \cdot\left\langle\mathbf{t}, a^{\prime}\right\rangle\right)=g\left(\langle a, \mathbf{t}\rangle \cdot\left\langle a^{\prime}, \mathbf{t}\right\rangle^{*}\right) \\
& =g(\langle a, \mathbf{t}\rangle) \cdot g\left(\left\langle a^{\prime}, \mathbf{t}\right\rangle\right)^{*}=\langle\tilde{g}(a), \mathbf{t}\rangle \cdot\left\langle\tilde{g}\left(a^{\prime}\right), \mathbf{t}\right\rangle^{*} \\
& =\langle\tilde{g}(a), \mathbf{t}\rangle \cdot\left\langle\mathbf{t}, \tilde{g}\left(a^{\prime}\right)\right\rangle=\left\langle\tilde{g}(a), \tilde{g}\left(a^{\prime}\right)\right\rangle .
\end{aligned}
$$

Thus, $g=S(\tilde{g})$, and the proof of surjectivity is complete.

Theorem 8.7. The variety of generalized Sugihara monoids and the variety of nuclear relative Stone algebras are categorically equivalent.

In particular, a category equivalence from NRSA to GSM is witnessed by the functor that sends $\boldsymbol{A}$ to $\boldsymbol{S}(\boldsymbol{A})$ and $h$ to $S(h)$ for all $\boldsymbol{A}, \boldsymbol{B} \in$ NRSA and all $h \in$ $\operatorname{Hom}(\boldsymbol{A}, \boldsymbol{B})$ (where $S(h)$ is as in Theorem 8.6).

The nuclear negative cone functor (sending $\boldsymbol{A}$ to $\boldsymbol{A}_{\diamond}^{-}$and $g$ to $\left.g\right|_{A^{-}}$for all $\boldsymbol{A}, \boldsymbol{B} \in \mathrm{GSM}$ and $g \in \operatorname{Hom}(\boldsymbol{A}, \boldsymbol{B}))$ is a reverse functor for $S$.

Proof. The first two assertions follow from Theorems 8.1, 8.5 and 8.6 (cf. items (ii) and (iii) in the first paragraph of Section 6). The last assertion follows easily from Theorems 8.2 and 8.5 .

\section{Categorical algebraic properties}

By Theorem 8.7, all purely categorical properties of NRSA will persist in GSM, and some of them are easier to establish in the integral setting of NRSA. We identify a number of properties of this kind.

Let $\mathrm{K}$ be a quasivariety of algebras. A congruence $\theta$ of an algebra $\boldsymbol{A}$ is called a $\mathrm{K}$-congruence if $\boldsymbol{A} / \theta \in \mathrm{K}$. We say that $\mathrm{K}$ has the relative congruence extension property if, for each $\boldsymbol{B} \in \mathrm{K}$, the $\mathrm{K}$-congruences of any subalgebra $\boldsymbol{A}$ of $\boldsymbol{B}$ are just the restrictions to $A \times A$ of the $\mathrm{K}$-congruences of $\boldsymbol{B}$. This reduces to the ordinary congruence extension property when $\mathrm{K}$ is a variety. 
Recall that a homomorphism $h$ between algebras in $\mathrm{K}$ is called a $(\mathrm{K}-)$ epimorphism provided that, for any two homomorphisms $f, g$ from the target of $h$ to a single member of $\mathrm{K}$, if $f \circ h=g \circ h$, then $f=g$. Clearly, every surjective homomorphism between algebras in $\mathrm{K}$ is an epimorphism, but the converse is not generally true. If every $\mathrm{K}$-epimorphism $h$ is surjective, then $\mathrm{K}$ is said to have the ES property. Note that, when verifying this property, we may assume without loss of generality that $h$ is an inclusion map.

The strong epimorphism-surjectivity (or strong ES) property for $\mathrm{K}$ asks that whenever $\boldsymbol{A}$ is a subalgebra of some $\boldsymbol{B} \in \mathrm{K}$ and $b \in B-A$, then there are two homomorphisms from $\boldsymbol{B}$ to a single member of $\mathrm{K}$ that agree on $A$ but not at $b$. This clearly implies the ES property. The weak ES property for $\mathrm{K}$ forbids non-surjective $\mathrm{K}$-epimorphisms $h: \boldsymbol{A} \longrightarrow \boldsymbol{B}$ in all cases where $\boldsymbol{B}$ is generated (as an algebra) by $X \cup h[A]$ for some finite $X \subseteq B$. It makes no difference to this definition if we stipulate that $X$ is a singleton.

The amalgamation property for a class $\mathrm{K}$ of similar algebras is the demand that, for any two embeddings $g_{B}: \boldsymbol{A} \longrightarrow \boldsymbol{B}$ and $g_{C}: \boldsymbol{A} \longrightarrow \boldsymbol{C}$ between algebras in K, there exist embeddings $f_{B}: \boldsymbol{B} \longrightarrow \boldsymbol{D}$ and $f_{C}: \boldsymbol{C} \longrightarrow \boldsymbol{D}$, with $\boldsymbol{D} \in \mathrm{K}$, such that $f_{B} \circ g_{B}=f_{C} \circ g_{C}$. The strong amalgamation property for $\mathrm{K}$ asks, in addition, that $\boldsymbol{D}, f_{B}$ and $f_{C}$ can be chosen so that $\left(f_{B} \circ g_{B}\right)[A]=f_{B}[B] \cap f_{C}[C]$.

These conditions are linked as follows (see [33, 37, 55] and [32, Sec. 2.5.3]).

Theorem 9.1. A quasivariety has the strong amalgamation property iff it has the amalgamation and weak ES properties. In that case, it also has the strong ES property.

Clearly, a category equivalence functor $F$ between quasivarieties sends monomorphisms (i.e., embeddings) to monomorphisms, and epimorphisms to epimorphisms. Less obviously, the same applies to surjective homomorphisms (see [44, p. 222], for instance). Thus, the amalgamation, ES and relative congruence extension properties are preserved by $F$. Consequently, strong amalgamation transfers as well, by Theorem 9.1. The strong and weak ES properties persist under $F$ too, even in the absence of amalgamation (see [26, Remark 5.10]).

We shall want to apply Theorem 8.7 in conjunction with the following general result, which derives from [5, 6] (see [26, Remark 7.2] for an explanation).

Theorem 9.2. Suppose $F: C \longrightarrow D$ witnesses a category equivalence between quasivarieties. Then, for each subquasivariety $\mathrm{E}$ of $\mathrm{C}$, the restriction of $F$ to $\mathrm{E}$ witnesses a category equivalence between the concrete categories $\mathrm{E}$ and

$$
\mathrm{E}^{\prime}:=\{\boldsymbol{B} \in \mathrm{D}: \boldsymbol{B} \cong F(\boldsymbol{A}) \text { for some } \boldsymbol{A} \in \mathrm{E}\},
$$

and the map $\mathrm{E} \mapsto \mathrm{E}^{\prime}$ defines an isomorphism between the subquasivariety lattices of $\mathrm{C}$ and $\mathrm{D}$, which takes the subvarieties of $\mathrm{C}$ onto those of $\mathrm{D}$.

Let $F$ be as in Theorem 9.2. If an algebra $\boldsymbol{A} \in \mathrm{C}$ possesses one of the following properties, then so does the algebra $F(\boldsymbol{A}) \in \mathrm{D}$ :

$\boldsymbol{A}$ is finite,

$\boldsymbol{A}$ is finitely generated.

This is explained in 44]. Consequently, if C is locally finite, then so is D. For example, Theorem 8.7 implies that NRSA is locally finite, although it is not hard to prove this directly. 
We have noted that $F$ preserves the injectivity and surjectivity of homomorphisms, and it sends products to products, as these are determined, up to isomorphism, by their categorical features. So, if $g$ is a subdirect embedding of $\boldsymbol{A}$ into $\prod_{i \in I} \boldsymbol{A}_{i}$, where $\boldsymbol{A}, \boldsymbol{A}_{i} \in \mathrm{C}$ for all $i \in I$, then $F(g)$ is a subdirect embedding of $F(\boldsymbol{A})$ into $\prod_{i \in I} F\left(\boldsymbol{A}_{i}\right)$. In particular, when $C$ and D are varieties, the list of $F$-invariant properties includes:

$$
\begin{aligned}
& \boldsymbol{A} \text { is finitely subdirectly irreducible, } \\
& \boldsymbol{A} \text { is subdirectly irreducible. }
\end{aligned}
$$

From Theorem 9.2 and the above remarks, we obtain:

Theorem 9.3. Let $F: \mathrm{C} \longrightarrow \mathrm{D}$ be a category equivalence between varieties. Let $\mathrm{X}$ be a subvariety of $\mathrm{C}$ and $\mathrm{Y}$ a subvariety of $\mathrm{D}$. Then $F$ restricts to a category equivalence from $\mathrm{X}$ to $\mathrm{Y}$ iff the [finitely] subdirectly irreducible algebras in $\mathrm{Y}$ are exactly the isomorphic copies of the F-images of the [finitely] subdirectly irreducible algebras in $\mathrm{X}$.

\section{Subvarieties AND EXPANSIONS OF GSM}

Definition 10.1. The variety CGSM of centred generalized Sugihara monoids consists of the algebras in GSM that satisfy

$$
\mathbf{t} \leq x^{* *} \vee\left(x^{* *} \rightarrow x\right) .
$$

The finitely subdirectly irreducible algebras in CGSM are exactly the totally ordered idempotent CRLs $\boldsymbol{A}$ such that, for all $a \in A$, we have

$$
a^{* *}=a \text { or } a^{*}=\mathbf{t}>a \text {. }
$$

In this case, in the notation of Theorem 3.2(ii), if $\mathbf{t} \leq b \in A$, then $\left|Y_{b}\right|=1$ or $Y_{b}=Y_{\mathbf{t}}$. The CRL-reduct of $\boldsymbol{Z}-\{0\}$ is therefore centred (with $Y_{1}=\{-1,1\}$ and $Y_{b}=\{-b\}$ for $\left.2 \leq b \in \mathbb{Z}\right)$, so PSM $\subseteq$ CGSM.

$$
\left\{\begin{array}{l}
b=b^{* *} \\
a=a^{* *} \\
\mathbf{t}=x^{*} \text { for all } x \in Y_{\mathbf{t}} \\
a^{*} \\
b^{*}
\end{array}\right.
$$

Figure 2. A totally ordered centred algebra in GSM

Theorems 8.7 and 9.2 show that CGSM and PSM are categorically equivalent to suitable subvarieties of NRSA. Let us identify those subvarieties. 
Lemma 10.2. The following conditions on an algebra $\boldsymbol{A} \in$ NRSA are equivalent.

(i) $\boldsymbol{A}$ satisfies $\diamond(\diamond x \rightarrow x)=\mathbf{t}$.

(ii) $\boldsymbol{A}$ satisfies $x \rightarrow \diamond y=\diamond(x \rightarrow y)$.

(iii) $\boldsymbol{A}$ satisfies $x \rightarrow \diamond y \leq \diamond(x \rightarrow y)$.

Proof. Assume without loss of generality that $\boldsymbol{A}$ is totally ordered. We make repeated use of (14).

(ii) $\Rightarrow$ (ii): Let $a, b \in A$. If $a \leq b$, then $a \leq \diamond b$ and

$$
a \rightarrow \diamond b=\mathbf{t}=\diamond \mathbf{t}=\diamond(a \rightarrow b) .
$$

Now assume $a>b$, so $a \rightarrow b=b$. If $a>\diamond b$, then $a \rightarrow \diamond b=\diamond b=\diamond(a \rightarrow b)$. And if $a \leq \diamond b$, then $\diamond b>b$, whence

$$
a \rightarrow \diamond b=\mathbf{t}=\diamond(\diamond b \rightarrow b)[\text { by (ii) }]=\diamond b=\diamond(a \rightarrow b) .
$$

(iii) $\Rightarrow$ (iii) is trivial.

(iii) $\Rightarrow$ (ii): Given $a \in A$, we have $\diamond(\diamond a \rightarrow a) \leq \mathbf{t}=\diamond a \rightarrow \diamond a \leq \diamond(\diamond a \rightarrow a)$, by (iii), so $\diamond(\diamond a \rightarrow a)=\mathbf{t}$.

Let $\mathrm{V}$ be the subvariety of NRSA axiomatized (relative to NRSA) by

$$
\diamond(\diamond x \rightarrow x)=\mathbf{t}
$$

By Theorem 7.1, the finitely subdirectly irreducible algebras in $\mathrm{V}$ are the totally ordered nuclear Brouwerian algebras $\boldsymbol{A}$ such that, for each $a \in A$, the element $\diamond^{\boldsymbol{A}} a$ is $a$ or t. In any such $\boldsymbol{A}$, the set

$$
F:=\left\{a \in A: \diamond^{A} a=\mathbf{t}\right\}
$$

is upward-closed, and $\nabla^{\boldsymbol{A}}$ is the identity function on the complement of $F$.

Theorem 10.3. CGSM is categorically equivalent to $\mathrm{V}$.

Proof. Let $\boldsymbol{A}$ be a generalized Sugihara monoid. Then $\boldsymbol{A} \cong \boldsymbol{S}\left(\boldsymbol{A}_{\diamond}^{-}\right)$, by Theorem 8.5. So, by the above remarks, $\boldsymbol{A}$ is a finitely subdirectly irreducible member of CGSM iff $\boldsymbol{A}_{\diamond}^{-}$is a finitely subdirectly irreducible member of $\mathrm{V}$, and the result follows from Theorem 9.3

Let $\mathrm{W}$ be the subvariety of NRSA axiomatized by

$$
(\diamond x \rightarrow x) \vee((y \vee(y \rightarrow x)) \wedge \diamond x)=\mathbf{t} .
$$

By Theorem 7.1, the finitely subdirectly irreducible algebras in $\mathrm{W}$ are the totally ordered nuclear Brouwerian algebras $\boldsymbol{A}$ such that

either (1) $\diamond^{\boldsymbol{A}}$ is the identity function of $A$, or $(2)\langle A ; \leq\rangle$ has a co-atom $c$ and $\diamond^{\boldsymbol{A}} c=\mathbf{t}$ and $\diamond^{\boldsymbol{A}} a=a$ whenever $c \neq a \in A$.

Consequently, $\mathrm{W} \subseteq \mathrm{V}$ and, if $\boldsymbol{B}$ denotes the CRL-reduct of $\boldsymbol{Z}-\{0\}$, then $\boldsymbol{B}_{\diamond}^{-} \in \mathrm{W}$.

Theorem 10.4. PSM is categorically equivalent to W.

Proof. Whenever $\boldsymbol{C}$ is a subdirectly irreducible algebra in the quasivariety generated by a class $\mathrm{Y}$ of similar algebras, then $\boldsymbol{C}$ can be embedded into an ultraproduct of members of Y.2 So, because PSM is the smallest quasivariety containing the CRL-reduct $\boldsymbol{B}$ of $\boldsymbol{Z}-\{0\}$, every subdirectly irreducible positive Sugihara monoid

\footnotetext{
2 This is well known. For a stronger result; see [16, Lem. 1.5].
} 
embeds into an ultrapower of $\boldsymbol{B}$. Theorems 8.7 and 9.2 show that PSM is categorically equivalent to $\mathrm{PSM}_{\diamond}^{-}$, and that $\mathrm{PSM}_{\diamond}^{-}$is a variety (because PSM is). We show that $\mathrm{PSM}_{\diamond}^{-}=\mathrm{W}$.

Let $\boldsymbol{A} \in \mathrm{PSM}$ and suppose $\boldsymbol{A}_{\diamond}^{-}$is finitely generated (hence finite). Then $\boldsymbol{A}$ is finite, because $\boldsymbol{A} \cong \boldsymbol{S}\left(\boldsymbol{A}_{\diamond}^{-}\right)$, by Theorem 8.5. Consequently, $\boldsymbol{A}$ is a subdirect product of finite algebras $\boldsymbol{A}_{i}(i \in I)$, each of which embeds into an ultrapower of $\boldsymbol{B}$. For each $i$, the property of not containing a subalgebra isomorphic to $\boldsymbol{A}_{i}$ persists in ultraproducts, as it is first order (owing to the finite size and type of $\boldsymbol{A}_{i}$ ). So, each $\boldsymbol{A}_{i}$ embeds into $\boldsymbol{B}$ itself. Because the nuclear negative cone functor is a category equivalence from GSM to NRSA, it follows that $\boldsymbol{A}_{\diamond}^{-}$is a subdirect product of the algebras $\left(\boldsymbol{A}_{i}\right)_{\diamond}^{-}, i \in I$, and that each $\left(\boldsymbol{A}_{i}\right)_{\diamond}^{-}$embeds into $\boldsymbol{B}_{\diamond}^{-}$, whence $\boldsymbol{A}_{\diamond}^{-} \in \mathrm{W}$ (because $\boldsymbol{B}_{\diamond}^{-} \in \mathrm{W}$ ). Therefore, $\mathrm{PSM}_{\diamond}^{-} \subseteq \mathrm{W}$, as every variety is generated by its finitely generated members.

Conversely, suppose $\boldsymbol{A} \in \mathrm{W}$. Since $\boldsymbol{A} \cong \boldsymbol{S}(\boldsymbol{A})_{\diamond}^{-}$(Theorem 8.2), it suffices to show that $\boldsymbol{S}(\boldsymbol{A}) \in$ PSM. Again, because $S$ is an equivalence functor, $\boldsymbol{S}(\boldsymbol{A})$ is a subdirect product of algebras $\boldsymbol{S}\left(\boldsymbol{A}_{i}\right)(i \in I)$, where each $\boldsymbol{A}_{i}$ is a subdirectly irreducible member of W. Thus, each $\boldsymbol{A}_{i}$ is a totally ordered Brouwerian algebra with a co-atom $c_{i}$, and $\nabla^{\boldsymbol{A}_{i}}$ fixes all elements of $A_{i}$ except possibly $c_{i}$ (in which case, $\left.\nabla^{\boldsymbol{A}_{i}} c_{i}=\mathbf{t}_{i}:=\mathbf{t}^{\boldsymbol{A}_{i}}\right)$. It follows that each $\boldsymbol{S}\left(\boldsymbol{A}_{i}\right)$ is a totally ordered member of GSM, and that the universe $S\left(\boldsymbol{A}_{i}\right)$ of $\boldsymbol{S}\left(\boldsymbol{A}_{i}\right)$ is

$$
X:=\left(A_{i} \times\left\{\mathbf{t}_{i}\right\}\right) \cup\left(\left\{\mathbf{t}_{i}\right\} \times A_{i}\right)
$$

if $\nabla^{\boldsymbol{A}_{i}}$ is the identity function on $A_{i}$; otherwise, $S\left(\boldsymbol{A}_{i}\right)=X-\left\{\left\langle\mathbf{t}_{i}, c_{i}\right\rangle\right\}$. It is then easy to see that every finitely generated-i.e., finite - subalgebra of each $\boldsymbol{S}\left(\boldsymbol{A}_{i}\right)$ embeds into $\boldsymbol{B}$ (cf. Example 8.4). Therefore, each $\boldsymbol{S}\left(\boldsymbol{A}_{i}\right)$ belongs to PSM, and so $\boldsymbol{S}(\boldsymbol{A}) \in$ PSM.

\section{Sugihara Monoids.}

Let $\mathrm{X}$ be the class of all $\langle\boldsymbol{A}, \mathbf{f}\rangle$, with $\boldsymbol{A} \in$ NRSA and $\mathbf{f} \in A$, satisfying

$$
\begin{aligned}
& x \vee(x \rightarrow \mathbf{f})=\mathbf{t}=\diamond(\diamond x \rightarrow x), \\
& \diamond x=\mathbf{t} \Longleftrightarrow \mathbf{f} \leq x .
\end{aligned}
$$

By considering subdirectly irreducible algebras, we can show that the equations

$$
\diamond \mathbf{f}=\mathbf{t} \text { and }(\diamond x \rightarrow x) \vee(\mathbf{f} \rightarrow x)=\mathbf{t}
$$

could replace (37) in the definition of $\mathrm{X}$, so $\mathrm{X}$ is a variety.

For $\boldsymbol{A} \in$ NRSA and $\mathbf{f} \in A$, the algebra $\langle\boldsymbol{A}, \mathbf{f}\rangle$ is a finitely subdirectly irreducible member of $\mathrm{X}$ iff it is totally ordered and

either (1) $\mathbf{f}=\mathbf{t}$ and $\diamond^{\boldsymbol{A}}$ is the identity function of $A$, or (2) $\mathbf{f}$ is

a co-atom of $\langle A ; \leq\rangle$ and $\nabla \mathbf{f}=\mathbf{t}$ and $\diamond a=a$ whenever $\mathbf{f} \neq a \in A$.

Thus, if $\langle\boldsymbol{A}, \mathbf{f}\rangle \in \mathrm{X}$, then $\boldsymbol{A} \in \mathrm{W}$.

Theorem 10.5. SM is categorically equivalent to $\mathrm{X}$.

Proof. Given $\langle\boldsymbol{A}, \mathbf{f}\rangle \in \mathrm{X}$, we define $\boldsymbol{S}(\langle\boldsymbol{A}, \mathbf{f}\rangle):=\langle\boldsymbol{S}(\boldsymbol{A}), \neg\rangle$, where

$$
\neg\left\langle a, a^{\prime}\right\rangle:=\left\langle a, a^{\prime}\right\rangle \rightarrow\langle\mathbf{f}, \mathbf{t}\rangle \text { for all }\left\langle a, a^{\prime}\right\rangle \in S(\boldsymbol{A}) .
$$

Note that $\boldsymbol{S}(\boldsymbol{A}) \in \mathrm{PSM}$, by the proof of Theorem 10.4. We claim that $\boldsymbol{S}(\langle\boldsymbol{A}, \mathbf{f}\rangle)$ satisfies $\neg \neg x=x$. As in the proof of Theorem 8.1. it suffices to verify this under the assumption that $\boldsymbol{A}$ is totally ordered, whence, for each $\left\langle a, a^{\prime}\right\rangle \in S(\boldsymbol{A})$, either 
$a$ or $a^{\prime}$ is $\mathbf{t}$, and $\nabla a^{\prime}=a^{\prime}$. It follows straightforwardly from the definition of $\rightarrow$ in $\boldsymbol{S}(\boldsymbol{A})$ that $\neg \neg\left\langle\mathbf{t}, a^{\prime}\right\rangle=\left\langle\mathbf{t}, a^{\prime}\right\rangle$ if $\diamond a^{\prime}=a^{\prime}$. To show that $\neg \neg\langle a, \mathbf{t}\rangle=\langle a, \mathbf{t}\rangle$, the reader should separate the cases $a<\mathbf{f}, a=\mathbf{f}, a=\mathbf{t}$. Thus, $\boldsymbol{S}(\langle\boldsymbol{A}, \mathbf{f}\rangle) \in \mathrm{SM}$.

Because $\neg$ is defined in terms of $\rightarrow$ on $\boldsymbol{S}(\langle\boldsymbol{A}, \mathbf{f}\rangle)$, Theorems 8.5 and 8.6 go through with $\mathrm{X}$ and $\mathrm{SM}$ in the respective roles of RSA and GSM. This gives an equivalence functor $S$ from $\mathrm{X}$ to $\mathrm{SM}$.

It is easily checked that a reverse functor $F: \mathrm{SM} \longrightarrow \mathrm{X}$ for $S$ is given by $F(\boldsymbol{A})=$ $\left\langle\boldsymbol{A}_{\diamond}^{-}, \neg \mathbf{t}\right\rangle$ and $F(h)=\left.h\right|_{A^{-}}$for all $\boldsymbol{A}, \boldsymbol{B} \in \mathrm{SM}$ and $h \in \operatorname{Hom}(\boldsymbol{A}, \boldsymbol{B})$.

If $\boldsymbol{A} \in \mathrm{RSA}$ is considered as a member of $\mathrm{X}$ satisfying $\diamond x=x$ and $\mathbf{f}=\mathbf{t}$, then (38) simplifies to

$$
\neg\left\langle a, a^{\prime}\right\rangle=\left\langle a^{\prime}, a\right\rangle \text { for all }\left\langle a, a^{\prime}\right\rangle \in S(\boldsymbol{A})
$$

and $\langle\boldsymbol{S}(\boldsymbol{A}), \neg\rangle$ is an odd Sugihara monoid. This recaptures the category equivalence between OSM and RSA established in [26].

The above results are summarized in Figure 3. The horizontal double lines signify category equivalence, while the solid single lines indicate containment. The broken single lines signify that the algebras in the lower variety are (or are termwise equivalent to) expansions of suitable algebras in the upper variety.

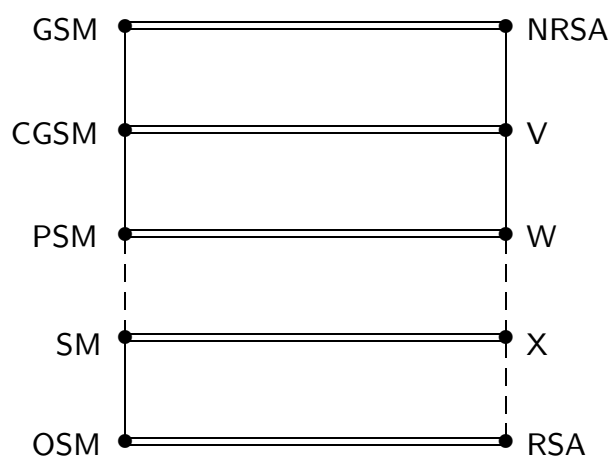

FiguRE 3. Summary of category equivalences

\section{Bounds.}

If a CRL $\boldsymbol{A}$ has a least element $\perp$, then $T=\perp \rightarrow \perp$ is its greatest element. In this case, the expansion $\boldsymbol{B}$ of $\boldsymbol{A}$ by the distinguished element $\perp$ is called a bounded $C R L$, and we sometimes write $\boldsymbol{B}=\boldsymbol{A}_{\perp}$. The negative cone $\boldsymbol{B}^{-}$of $\boldsymbol{B}$ is defined as before, except that $\perp^{\boldsymbol{B}}$ is distinguished in $\boldsymbol{B}^{-}$. If $\boldsymbol{C}$ is a class of CRLs, then $C_{\perp}$ shall denote the class of all bounded CRLs whose CRL-reducts belong to $C$. We extend these conventions to involutive and nuclear CRLs in the obvious way. We also extend the notation NC to bounded classes C.

Let GA denote the variety of bounded relative Stone algebras, a.k.a. Gödel algebras. The category equivalence between GSM and NRSA can be extended to one between $\mathrm{GSM}_{\perp}$ and NGA. In the construction of $\boldsymbol{S}(\boldsymbol{A})$, we simply define $\perp^{\boldsymbol{S}(\boldsymbol{A})}=\left\langle\perp^{\boldsymbol{A}}, \mathbf{t}\right\rangle$ for $\boldsymbol{A} \in \mathrm{NGA}$. Similarly, $\mathrm{CGSM}_{\perp}, \mathrm{PSM}_{\perp}, \mathrm{SM}_{\perp}$ and $\mathrm{OSM}_{\perp}$ are categorically equivalent to $\mathrm{V}_{\perp}, \mathrm{W}_{\perp}, \mathrm{X}_{\perp}$ and $\mathrm{GA}$, respectively. 


\section{Deductive Systems}

For present purposes, a deductive system is any substitution-invariant finitary consequence relation $\vdash$ over formulas in an algebraic language. In this context, basic operation symbols and terms are usually called connectives and formulas, respectively. Substitutions are the homomorphisms between formula algebras, i.e., between absolutely free algebras generated by sets of variables of the language. Thus, the defining postulates of deductive systems are as follows, where $\Gamma \cup \Pi \cup\{s\}$ is any set of formulas:

$$
\begin{aligned}
& \text { if } s \in \Gamma \text {, then } \Gamma \vdash s \text {; } \\
& \text { if } \Gamma \vdash t \text { for all } t \in \Pi \text {, and } \Pi \vdash s \text {, then } \Gamma \vdash s \text {; } \\
& \text { if } \Gamma \vdash s \text {, then } h[\Gamma] \vdash h(s) \text { for every substitution } h \text {; } \\
& \text { if } \Gamma \vdash s \text {, then } \Gamma^{\prime} \vdash s \text { for some finite } \Gamma^{\prime} \subseteq \Gamma \text { (finitarity). }
\end{aligned}
$$

The theorems of $\vdash$ are the formulas $s$ such that $\emptyset \vdash s$ (briefly, $\vdash s$ ). We signify ' $\Gamma \vdash t$ for all $t \in \Pi$ ' by $\Gamma \vdash \Pi$, and ' $\Gamma \vdash \Pi$ and $\Pi \vdash \Gamma$ ' by $\Gamma \dashv \vdash$. Also, $\Gamma, r \vdash s$ means $\Gamma \cup\{r\} \vdash s$. Deductive systems can be characterized as the natural deducibility relations $\vdash_{\mathbf{F}}$ of arbitrary formal systems $\mathbf{F}$ consisting of axioms and finite inference rules [39].

Example 11.1. For each quasivariety $K$ of CRL-expansions, we define a binary relation $\vdash_{K}$ from sets of formulas to single formulas as follows:

$$
\Gamma \vdash_{\mathrm{K}} s \text { iff, for some finite } \Gamma^{\prime} \subseteq \Gamma \text {, the quasi-equation }
$$

$$
\left(\&_{r \in \Gamma^{\prime}} \mathbf{t} \leq r(\bar{x})\right) \Longrightarrow \mathbf{t} \leq s(\bar{x})
$$

is valid in $\mathrm{K}$.

Here, \& denotes first order conjunction. Many familiar non-classical logics are specified by formal systems $\mathbf{F}$, where $\vdash_{\mathbf{F}}$ is $\vdash_{\mathbf{K}}$ for some such $\mathrm{K}$. For example, $\mathbf{R M}^{\mathbf{t}}$ and IUML correspond in this way to the varieties $S M$ and $O S M_{\perp}$, respectively (see [2, 18, 45]), while exponential-free linear logic corresponds to the variety of all bounded involutive CRLs (see [4, 27, 57]). Since CRLs satisfy (11), $\vdash_{\mathrm{K}}$ is always an algebraizable deductive system in the sense of [11, with $\mathrm{K}$ as its equivalent algebraic semantics. This allows us to apply 'bridge theorems' such as those in the next result.

Theorem 11.2. Let $\mathrm{K}$ be a [quasi] variety that is the equivalent algebraic semantics for a deductive system $\vdash$.

(i) $([10]) \vdash$ has a local deduction theorem iff $\mathrm{K}$ has the [relative] congruence extension property.

(ii) (9]) $\vdash$ has the infinite Beth definability property iff all epimorphisms between algebras in $\mathrm{K}$ are surjective.

(iii) $(9]) \vdash$ has the finite Beth property iff $\mathrm{K}$ has the weak epimorphismsurjectivity property.

(iv) ([31]) $\vdash$ has the projective Beth property iff $\mathrm{K}$ has the strong epimorphismsurjectivity property.

(v) ([17]) When the conditions in (ii) hold, then $\vdash$ has the interpolation property iff $\mathrm{K}$ has the amalgamation property.

The metalogical notions in Theorem 11.2 are defined below. 3

\footnotetext{
${ }^{3}$ Items (ii)-(V) of Theorem 11.2 appear in their full generality in the sources cited, but they were first established in more concrete settings. For accounts of their antecedents; see Czelakowski
} 
Definition 11.3. Let $\vdash$ be a deductive system.

(i) $\vdash$ has the interpolation property if the following is true: whenever $\Gamma \vdash s$, then $\Gamma \vdash \Gamma^{\prime}$ and $\Gamma^{\prime} \vdash s$ for some set $\Gamma^{\prime}$ of formulas, where every variable occurring in a formula from $\Gamma^{\prime}$ already occurs both in $s$ and in some formula from $\Gamma$ (unless $\Gamma$ and $s$ have no common variable).

(ii) $([14]) \vdash$ has a local deduction (-detachment) theorem if there is a family $\left\{\Lambda_{i}: i \in I\right\}$ of sets of binary formulas such that the rule

$$
\Gamma, r \vdash s \text { iff there exists } i \in I \text { such that }\left(\Gamma \vdash \ell(r, s) \text { for all } \ell \in \Lambda_{i}\right)
$$

applies to all sets of formulas $\Gamma \cup\{r, s\}$. The word 'local' is dropped if we can arrange that $|I|=1$.

Example 11.4 (cf. [25]). Let $\mathrm{K}$ be a variety of [involutive] [bounded] CRLs. Then $\vdash_{\mathrm{K}}$ has the following local deduction theorem:

$$
\Gamma, r \vdash_{\mathrm{K}} s \text { iff there exists } n \in \omega \text { such that } \Gamma \vdash_{\mathrm{K}}(r \wedge \mathbf{t})^{n} \rightarrow s .
$$

Here, $x^{0}:=\mathbf{t}$ and $x^{n+1}:=x^{n} \cdot x$ for each $n \in \omega$. If $\mathrm{K}$ satisfies $(x \wedge \mathbf{t})^{2}=x \wedge \mathbf{t}$, then (39) becomes a deduction theorem:

$$
\Gamma, r \vdash_{\mathrm{K}} s \text { iff } \Gamma \vdash_{\mathrm{K}}(r \wedge \mathbf{t}) \rightarrow s .
$$

It reduces to the classical deduction theorem

$$
\Gamma, r \vdash_{\mathrm{K}} s \text { iff } \Gamma \vdash_{\mathrm{K}} r \rightarrow s
$$

when the algebras in $\mathrm{K}$ are integral and idempotent. In (40), the implication from right to left does not depend on any special assumptions about $\mathrm{K}$.

We continue to use $x, y, z$, with or without indices, to denote variables. If $X$ is a set of variables, then $F m(X)$ denotes the set of all formulas involving only variables from $X$.

From now on, our deductive systems $\vdash$ will be assumed equivalential in the sense of [15, 52], i.e., there is a set $\Delta$ of binary formulas such that

$$
\begin{aligned}
& \vdash \Delta(x, x) \quad(\text { i.e., } \vdash d(x, x) \text { for all } d \in \Delta) \\
& \{x\} \cup \Delta(x, y) \vdash y \\
& \bigcup_{i=1}^{n} \Delta\left(x_{i}, y_{i}\right) \vdash \Delta\left(r\left(x_{1}, \ldots, x_{n}\right), r\left(y_{1}, \ldots, y_{n}\right)\right)
\end{aligned}
$$

for every connective $r$, where $n$ is the rank of $r$. Any such $\Delta$ is essentially unique, i.e., if $\Delta^{\prime}$ serves the same purpose, then $\Delta(x, y) \dashv \Delta^{\prime}(x, y)$. All algebraizable systems are equivalential [11. For the systems $\vdash_{\mathrm{K}}$ in Example 11.1, we can take $\Delta$ to be $\{x \rightarrow y, y \rightarrow x\}$, or alternatively $\{x \leftrightarrow y\}$.

Definition 11.5. The following terminology applies if $X, Y$ and $Z$ are disjoint sets of variables, where $X \neq \emptyset$ or the language contains some constant symbols.

Let $\Gamma \subseteq F m(X \cup Y \cup Z)$. We say that $\Gamma$ implicitly defines $Z$ in terms of $X$ via $Y$ in $\vdash$ provided that, for every $z \in Z$ and every substitution $h$, defined on $X \cup Y \cup Z$, if $h(x)=x$ for all $x \in X$, then

$$
\Gamma \cup h[\Gamma] \vdash \Delta(z, h(z)) .
$$

and Pigozzi [17, Gabbay and Maksimova [21, Hoogland [32], and Kihara and Ono [36]. In particular, (iii) was proved in a restricted form by I. Nemeti in [30. Thm. 5.6.10]. 
In the event that $Y=\emptyset$, we simply say that $\Gamma$ implicitly defines $Z$ in terms of $X$. On the other hand, we say that $\Gamma$ explicitly defines $Z$ in terms of $X$ via $Y$ in $\vdash$ provided that, for each $z \in Z$, there exists $t_{z} \in F m(X)$ such that

$$
\Gamma \vdash \Delta\left(z, t_{z}\right) .
$$

Again, we omit 'via $Y$ ' if $Y=\emptyset$.

Example 11.6. In classical propositional logic (CPL), the set

$$
\Gamma:=\left\{z \rightarrow x_{1}, z \rightarrow x_{2}, x_{1} \rightarrow\left(x_{2} \rightarrow z\right)\right\}
$$

implicitly defines $\{z\}$ in terms of $\left\{x_{1}, x_{2}\right\}$. It does so explicitly as well, because $\Gamma \vdash_{\mathbf{C P L}} z \leftrightarrow\left(x_{1} \wedge x_{2}\right)$. In the implication fragment of CPL, however, $\Gamma$ still defines $\{z\}$ implicitly in terms of $\left\{x_{1}, x_{2}\right\}$, but there is demonstrably no explicit definition (see [9]).

\section{Definition 11.7.}

(i) $([9]) \vdash$ has the infinite Beth (definability) property provided that, in $\vdash$, whenever $\Gamma \subseteq F m(X \cup Z)$ implicitly defines $Z$ in terms of $X$, then $\Gamma$ also explicitly defines $Z$ in terms of $X$.4

(ii) The finite Beth property is defined like the infinite one, except that $Z$ is required to be finite in the definition.

(iii) (cf. [8, p.76]) $\vdash$ has the projective Beth property provided that, in $\vdash$, whenever $\Gamma \subseteq F m(X \cup Y \cup Z)$ implicitly defines $Z$ in terms of $X$ via $Y$, then $\Gamma$ also explicitly defines $Z$ in terms of $X$ via $Y$.

In the definitions of the finite and projective Beth properties, it makes no difference if we stipulate that $Z$ is a singleton (see [9, 31]). According to [9, it is not known whether the finite Beth property implies the infinite one, but the latter is strictly weaker than the projective property.

\section{BROUWERIAN EXPANSIONS}

Our category equivalences reduce a number of questions about non-integral structures to questions about (integral) Brouwerian algebras and their expansions. In this section, therefore, we gather some facts about the latter. The following observation is well known.

Lemma 12.1. Let $\boldsymbol{A}$ be a Brouwerian algebra with $a_{i}, b_{i}, c, d \in A$, for $i=1, \ldots, n$, and let $\theta$ be the congruence of $\boldsymbol{A}$ generated by $\left\{\left\langle a_{i}, b_{i}\right\rangle: i=1, \ldots, n\right\}$. Then $c \equiv_{\theta} d$ iff $\bigwedge_{i=1}^{n} a_{i} \leftrightarrow b_{i} \leq c \leftrightarrow d$.

Recall the definition of $\vdash_{K}$ from Example 11.1. Note that when $\mathrm{K}$ consists of integral algebras and $\Gamma$ is finite, then $\Gamma \vdash_{\mathrm{K}} s$ iff $\mathrm{K}$ satisfies

$$
\left(\&_{r \in \Gamma} r=\mathbf{t}\right) \Longrightarrow s=\mathbf{t} \text {. }
$$

\footnotetext{
4 There is a subtlety here. The meaning of a Beth property should not depend on the number of variables of $\vdash$, otherwise Theorems 11.2 iii - iv could not hold. But the cardinality of a set of variables constrains the options for $\Gamma$ and the meaning of implicit definability. The problem is resolved by assuming that the variables of $\vdash$ form a proper class $V$, and that $\vdash$ is really a family of relations - one for each infinite subset of $V$; see [9] 17]. (Because $\vdash$ is finitary, it can be recovered from any one of these relations.) This explains why substitutions have been defined here as homomorphisms between formula algebras, rather than endomorphisms of a single algebra.
} 
Lemma 12.2. Let $\mathrm{K}$ be a variety of enriched Brouwerian algebras. Then the classical deduction theorem (40) holds for $\vdash_{\mathrm{K}}$ iff every member of $\mathrm{K}$ has the same congruences as its Brouwerian algebra reduct.

Proof. $(\Rightarrow)$ Let $f$ be an $n$-ary basic operation symbol of K. By (13),

$$
x_{1} \leftrightarrow y_{1}, \ldots, x_{n} \leftrightarrow y_{n} \vdash_{\mathrm{K}} f\left(x_{1}, \ldots, x_{n}\right) \leftrightarrow f\left(y_{1}, \ldots, y_{n}\right) .
$$

So, the deduction theorem and the Brouwerian identity

$$
x \rightarrow(y \rightarrow z)=(x \wedge y) \rightarrow z
$$

yield $\vdash_{\mathrm{K}}\left(\bigwedge_{i=1}^{n} x_{i} \leftrightarrow y_{i}\right) \rightarrow\left(f\left(x_{1}, \ldots, x_{n}\right) \leftrightarrow f\left(y_{1}, \ldots, y_{n}\right)\right)$, i.e., K satisfies

$$
\bigwedge_{i=1}^{n} x_{i} \leftrightarrow y_{i} \leq f\left(x_{1}, \ldots, x_{n}\right) \leftrightarrow f\left(y_{1}, \ldots, y_{n}\right) .
$$

Let $\theta$ be a congruence of the Brouwerian reduct $\boldsymbol{B}$ of some $\boldsymbol{A} \in \mathrm{K}$, and suppose $a_{i} \equiv_{\theta} b_{i}$ for $i=1, \ldots, n$. Then $f^{\boldsymbol{A}}\left(a_{1}, \ldots, a_{n}\right) \equiv_{\theta} f^{\boldsymbol{A}}\left(b_{1}, \ldots, b_{n}\right)$, by (42) and Lemma 12.1

$(\Leftarrow)$ Suppose $\Gamma, r^{\prime} \vdash_{\mathrm{K}} s$, where, without loss of generality, $\Gamma$ is finite. Let $r$ be the $\wedge$-conjunction of $r^{\prime}$ and the elements of $\Gamma$, so $r \vdash_{\mathrm{K}} s$, i.e., $\mathrm{K}$ satisfies $r=\mathbf{t} \Longrightarrow$ $s=\mathbf{t}$. We aim to show that $\vdash_{\mathrm{K}} r \rightarrow s$, as this, with (41), yields $\Gamma \vdash_{\mathrm{K}} r^{\prime} \rightarrow s$. Let $\boldsymbol{A} \in \mathrm{K}$ and let $h$ be a homomorphism from a formula algebra for $\vdash_{\mathrm{K}}$ into $\boldsymbol{A}$. Then $\langle h(s), \mathbf{t}\rangle$ belongs to the congruence of $\boldsymbol{A}$ generated by $\langle h(r), \mathbf{t}\rangle$, because $\mathrm{K}$ is closed under homomorphic images. So, by assumption, $\langle h(s), \mathbf{t}\rangle$ belongs to the congruence of $\boldsymbol{B}$ generated by $\langle h(r), \mathbf{t}\rangle$, where $\boldsymbol{B}$ is the Brouwerian reduct of $\boldsymbol{A}$. Then, by Lemma 12.1 $h(r) \leq h(s)$, i.e., $h(r \rightarrow s)=\mathbf{t}$. Since $\boldsymbol{A}$ and $h$ were arbitrary, this shows that $\vdash_{\mathrm{K}} r \rightarrow s$.

The next theorem generalizes an argument of G. Kreisel [38] straightforwardly. It will be important in the sequel, so we include a proof.

Theorem 12.3. Let $\mathrm{K}$ be a variety of enriched Brouwerian algebras, where every member of $\mathrm{K}$ has the same congruences as its Brouwerian algebra reduct. Then $\vdash_{\mathrm{K}}$ has the finite Beth property.

Proof. We abbreviate $\vdash_{\mathrm{K}}$ as $\vdash$. The classical deduction theorem holds for $\vdash$, by Lemma 12.2, Let $X \cup\{z\}$ be a set of variables, with $z \notin X$, and suppose $\Gamma \subseteq F m(X \cup\{z\})$ defines $\{z\}$ implicitly in terms of $X$ in $\vdash$. By the remark after Definition 11.7(iii), it suffices to show that $\Gamma$ defines $\{z\}$ explicitly in terms of $X$ in $\vdash$. Choose a substitution $h$ such that $h(x)=x$ for all $x \in X$ and $h(z)=\mathbf{t}$. By assumption, $\Gamma \cup h[\Gamma] \vdash z \leftrightarrow \mathbf{t}$, hence $\Gamma \cup h[\Gamma] \vdash z$. Since $\vdash$ is finitary, there is a finite subset $\Gamma^{\prime}=\left\{r_{1}, \ldots, r_{n}\right\}$ of $\Gamma$ such that $\Gamma^{\prime} \cup h\left[\Gamma^{\prime}\right] \vdash z$. Let $r$ be the formula $r_{1} \wedge \cdots \wedge r_{n}$, so $h(r) \in F m(X)$, by definition of $h$, and $\Gamma \vdash r$ and $r, h(r) \vdash z$. Then $r \vdash h(r) \rightarrow z$, by the deduction theorem. On the other hand, $\mathrm{K}$ satisfies the quasi-equation

$$
(r=\mathbf{t} \& z=\mathbf{t}) \Longrightarrow h(r)=\mathbf{t},
$$

by definition of $h$, so $r, z \vdash h(r)$. By the deduction theorem, $r \vdash z \rightarrow h(r)$, so $r \vdash z \leftrightarrow h(r)$, whence $\Gamma \vdash z \leftrightarrow h(r)$.

A Heyting algebra is a bounded Brouwerian algebra. Let BrA and HA denote the varieties of Brouwerian and Heyting algebras, respectively. For any class $\mathrm{K}$ of CRL-expansions, let $\mathrm{K}_{\mathbf{f}}$ be the class of all algebras $\langle\boldsymbol{A}, \mathbf{f}\rangle$ such that $\boldsymbol{A} \in \mathrm{K}$ and $\mathbf{f} \in A$. 
Lemma 12.4. Let $\boldsymbol{A}$ be an algebra in one of the following varieties: $\mathrm{NBrA}, \mathrm{NBrA}_{\mathbf{f}}$, $\mathrm{NHA}, \mathrm{NHA}_{\mathbf{f}}$. Then $\boldsymbol{A}$ has the same congruences as its Brouwerian algebra reduct.

Proof. The case of NBrA is covered by Theorem 17.1, In the other cases, $\boldsymbol{A}$ is an expansion by constants only of some algebra in NBrA, so the result follows.

Corollary 12.5. Let $\mathrm{K}$ be a subvariety of one of the following varieties: NBrA, $\mathrm{NBrA}_{\mathbf{f}}, \mathrm{NHA}, \mathrm{NHA}_{\mathbf{f}}$. Then $\mathrm{K}$ has the weak ES property.

Proof. This follows from Theorems 11.2(iii) and 12.3, and by Lemma 12.4.

Theorem 12.6 (Maksimova). Let $\mathrm{K}$ be a variety of enriched Brouwerian algebras, where every member of $\mathrm{K}$ has the same congruences as its Brouwerian algebra reduct. Then the following conditions are equivalent:

(i) $\mathrm{K}$ has the amalgamation property.

(ii) The class of finitely subdirectly irreducible algebras in $\mathrm{K}$ has the amalgamation property.

This is shown in Chapter 6 of [21, without the provision for extra operations. The proof needs no modification, however, in view of the additional assumptions, Lemma 12.2 and the characterization of finitely subdirectly irreducible algebras in Section 3. The result below is derived as a corollary in [21]. Both theorems originate in [40,41].

Theorem 12.7. The variety RSA of relative Stone algebras and the variety GA of Gödel algebras have the amalgamation property.

Suppose $\boldsymbol{A}$ and $\boldsymbol{B}$ are totally ordered Brouwerian algebras with a common greatest element $\mathbf{t}$, where $A \cap B=\{\mathbf{t}\}$. The ordinal sum $\boldsymbol{A} \oplus \boldsymbol{B}$ is the unique totally ordered Brouwerian algebra with universe $A \cup B$ such that the order $\leq$ of $\boldsymbol{A} \oplus \boldsymbol{B}$ restricts to the original order of $\boldsymbol{A}$ and to that of $\boldsymbol{B}$, while $a<b$ whenever $\mathbf{t} \neq a \in A$ and $b \in B$. The same construction produces a Gödel algebra when $\boldsymbol{A}$ is bounded, regardless of whether $\boldsymbol{B}$ is bounded.

Lemma 12.8. The varieties $\mathrm{V}, \mathrm{W}$ and $\mathrm{X}$ from Section 10 have the amalgamation property, and so do $\mathrm{V}_{\perp}, \mathrm{W}_{\perp}$ and $\mathrm{X}_{\perp}$.

Proof. We deal with $\mathrm{V}$ first. Let $\overline{\mathrm{V}}$ be the class of all totally ordered (i.e., finitely subdirectly irreducible) algebras in $\mathrm{V}$. Let $\boldsymbol{A}_{1}, \boldsymbol{A}_{2} \in \overline{\mathrm{V}}$, and let $\boldsymbol{A}_{0}$ be a subalgebra both of $\boldsymbol{A}_{1}$ and of $\boldsymbol{A}_{2}$. By Theorem 12.6, we need only show that there exist $\boldsymbol{B} \in \overline{\mathrm{V}}$ and embeddings $h_{1}: \boldsymbol{A}_{1} \longrightarrow \boldsymbol{B}$ and $h_{2}: \boldsymbol{A}_{2} \longrightarrow \boldsymbol{B}$, with $\left.h_{1}\right|_{A_{0}}=\left.h_{2}\right|_{A_{0}}$. We may assume without loss of generality that $A_{0}=A_{1} \cap A_{2}$.

Given $i \in\{0,1,2\}$, the sets

$$
A_{i}^{\prime \prime}:=\left\{a \in A_{i}: \diamond a=\mathbf{t}\right\} \text { and } A_{i}^{\prime}:=\left(A_{i}-A_{i}^{\prime \prime}\right) \cup\{\mathbf{t}\}
$$

are subuniverses of $\boldsymbol{A}_{i}$ and, because $\boldsymbol{A}_{0}$ is a subalgebra of $\boldsymbol{A}_{i}$, we have

$$
A_{0}^{\prime}=A_{1}^{\prime} \cap A_{2}^{\prime} \text { and } A_{0}^{\prime \prime}=A_{1}^{\prime \prime} \cap A_{2}^{\prime \prime} \text {. }
$$

Let $\boldsymbol{A}_{i}^{\prime}$ and $\boldsymbol{A}_{i}^{\prime \prime}$ be the RSA-subreducts of $\boldsymbol{A}_{i}$ whose universes are $A_{i}^{\prime}$ and $A_{i}^{\prime \prime}$, respectively. By Theorems 12.6 and 12.7, there exist totally ordered algebras $\boldsymbol{B}^{\prime}, \boldsymbol{B}^{\prime \prime} \in$ RSA such that, for each $i \in\{1,2\}$, there are embeddings $g_{i}^{\prime}: \boldsymbol{A}_{i}^{\prime} \longrightarrow \boldsymbol{B}^{\prime}$ and $g_{i}^{\prime \prime}: \boldsymbol{A}_{i}^{\prime \prime} \longrightarrow \boldsymbol{B}^{\prime \prime}$ with $\left.g_{1}^{\prime}\right|_{A_{0}^{\prime}}=\left.g_{2}^{\prime}\right|_{A_{0}^{\prime}}$ and $\left.g_{1}^{\prime \prime}\right|_{A_{0}^{\prime \prime}}=\left.g_{2}^{\prime \prime}\right|_{A_{0}^{\prime \prime}}$. Let $\boldsymbol{B}=\boldsymbol{B}^{\prime} \oplus \boldsymbol{B}^{\prime \prime}$, so $\boldsymbol{B} \in \mathrm{RSA}$, and $\boldsymbol{B}$ is totally ordered. Let $\boldsymbol{B}^{\diamond} \in$ NRSA be the expansion of $\boldsymbol{B}$ in which 
$\diamond b=b$ for all $b \in B^{\prime}$ and $\diamond b=\mathbf{t}$ for all $b \in B^{\prime \prime}$, so $\boldsymbol{B}^{\diamond} \in \overline{\mathrm{V}}$. For each $i \in\{1,2\}$, the relation $h_{i}=g_{i}^{\prime} \cup g_{i}^{\prime \prime}$ is a function embedding $\boldsymbol{A}_{i}$ into $\boldsymbol{B}^{\diamond}$, and $\left.h_{1}\right|_{A_{0}}=\left.h_{2}\right|_{A_{0}}$. This completes the proof in the case of $\mathrm{V}$.

In the case where $\mathrm{W}$ replaces $\mathrm{V}$, the argument needs modification. In an algebra from $\bar{W}$, if $\diamond$ maps an element other than $\mathbf{t}$ to $\mathbf{t}$, then that element must be a co-atom of the algebra. However, the algebra need not have a co-atom, and $\diamond$ need not map a co-atom to $\mathbf{t}$. Because $\boldsymbol{A}_{0}$ is a subalgebra of $\boldsymbol{A}_{1}$ and of $\boldsymbol{A}_{2}$, we must have $1 \leq\left|A_{0}^{\prime \prime}\right| \leq\left|A_{1}^{\prime \prime}\right|,\left|A_{2}^{\prime \prime}\right| \leq 2$, and if $\left|A_{0}^{\prime \prime}\right|=2$, then $A_{0}^{\prime \prime}=A_{1}^{\prime \prime}=A_{2}^{\prime \prime}$. If $A_{1}^{\prime \prime}=A_{2}^{\prime \prime}$, we replace $\boldsymbol{B}^{\prime \prime}$ by $\boldsymbol{A}_{1}^{\prime \prime}$ and $g_{i}^{\prime \prime}$ by the identity automorphism of $\boldsymbol{A}_{1}^{\prime \prime}$ for each $i \in\{1,2\}$. If $\left|A_{1}^{\prime \prime}\right|=2$ and $\left|A_{2}^{\prime \prime}\right|=1$, replace $\boldsymbol{B}^{\prime \prime}$ by $\boldsymbol{A}_{1}^{\prime \prime}$ and $g_{1}^{\prime \prime}$ by the identity function and $g_{2}^{\prime \prime}$ by the inclusion map. We proceed symmetrically if the roles of 1 and 2 are reversed. Finally, suppose $A_{1}^{\prime \prime}=\left\{a_{1}, \mathbf{t}\right\}$ and $A_{2}^{\prime \prime}=\left\{a_{2}, \mathbf{t}\right\}$, where the elements $a_{1}, a_{2}, \mathbf{t}$ are distinct, hence $a_{1}, a_{2} \notin A_{0}$. In this case, replace $\boldsymbol{B}^{\prime \prime}$ by $\boldsymbol{A}_{1}^{\prime \prime}$ and $g_{1}^{\prime \prime}$ by the identity function and $g_{2}^{\prime \prime}$ by the isomorphism that sends $a_{2}$ to $a_{1}$. These amendments ensure that $\boldsymbol{B}^{\diamond} \in \overline{\mathrm{W}}$, without compromising the amalgamation process.

Now consider the case where $\mathbf{X}$ replaces $\mathbf{W}$. An algebra from $\bar{X}$ is an $\mathbf{f}$-expansion of one from $\overline{\mathrm{W}}$, and if $\mathbf{f} \neq \mathbf{t}$, then $\mathbf{f}$ is a co-atom with $\diamond \mathbf{f}=\mathbf{t}$. Because $\boldsymbol{A}_{0}$ is a subalgebra of $\boldsymbol{A}_{1}$ and of $\boldsymbol{A}_{2}$, the identity $\mathbf{f}=\mathbf{t}$ holds in all three algebras or in none of them. Also, $A_{0}^{\prime \prime}$ already contains the $\boldsymbol{A}_{0}$-interpretations of both $\mathbf{f}$ and $\mathbf{t}$, and it has no other element. Consequently, no modification of the $\mathrm{W}$-approach is needed, although some cases disappear. The $\boldsymbol{B}^{\diamond}$-value of $\mathbf{f}$ is defined as the $g_{1}^{\prime \prime}$-image of the $\boldsymbol{A}_{0}$-value of $\mathbf{f}$.

The entire argument can be carried out in the bounded case, with no essential modification. Because the algebras $\boldsymbol{A}_{i}^{\prime}$ are bounded, the amalgamation property for GA (Theorem 12.7) is applied to them, but the $\boldsymbol{A}_{i}^{\prime \prime}$ are still amalgamated in RSA, before the ordinal sum is constructed.

Theorem 12.9. Every subquasivariety of $\mathrm{V}$ is a variety.

Proof. The argument is well known, except for the involvement of $\diamond$. Let $Q$ be a subquasivariety of $\mathrm{V}$, and let $h: \boldsymbol{A} \longrightarrow \boldsymbol{B}$ be a surjective homomorphism, with $\boldsymbol{A} \in \mathrm{Q}$. We must show that $\boldsymbol{B} \in \mathrm{Q}$. Every quasivariety is axiomatized by quasiequations (of finite length), so it suffices to show that every finitely generated subalgebra of $\boldsymbol{B}$ belongs to $\mathrm{Q}$. Any such subalgebra is the image under $h$ of a finitely generated subalgebra of $\boldsymbol{A}$, and $\mathrm{Q}$ is closed under subalgebras, so we may assume without loss of generality that $\boldsymbol{A}$ is finitely generated. But NRSA is a locally finite variety containing $\mathrm{V}$, so $\boldsymbol{A}$ is finite.

Let $\theta=\operatorname{ker} h$ (the congruence kernel of $h$ ). By Theorem 7.1 , the congruences of $\boldsymbol{A}$ are just those of the $\diamond$-free reduct of $\boldsymbol{A}$. This reduct is a relative Stone algebra, so $\mathbf{t} / \theta$ is a filter of the lattice $\langle A ; \wedge, \vee\rangle$. Since $A$ is finite, $\mathbf{t} / \theta$ has a least element, $\ell$ say. Define $g: A \longrightarrow A$ by $g(a)=\ell \rightarrow a(a \in A)$. Clearly, $g$ preserves $\wedge$ and $\mathbf{t}$. Integrality and contraction can be used to show that $g$ preserves $\rightarrow$, so it preserves $\checkmark$ as well, because RSA satisfies

$$
x \vee y=((x \rightarrow y) \rightarrow y) \wedge((y \rightarrow x) \rightarrow x) .
$$

Furthermore, $g$ preserves $\diamond$, because $\ell \rightarrow \diamond a=\diamond(\ell \rightarrow a)$ for all $a \in A$, by Lemma 10.2 and the definition of $\mathrm{V}$. Thus, $g$ is an endomorphism of $\boldsymbol{A}$, so its image $g[\boldsymbol{A}]$ is a subalgebra of $\boldsymbol{A}$, hence $g[\boldsymbol{A}] \in \mathrm{Q}$. For each $a \in A$, we have

$$
a \in \mathbf{t} / \operatorname{ker} g \text { iff } \ell \rightarrow a=\mathbf{t} \text { iff } \ell \leq a \text { iff } a \in \mathbf{t} / \theta \text {, }
$$


so $\mathbf{t} / \operatorname{ker} g=\mathbf{t} / \theta$. Since $\mathrm{V}$ is a $\mathbf{t}-$ regular variety, it follows that $\operatorname{ker} g=\theta$. By the Homomorphism Theorem, $\boldsymbol{B} \cong \boldsymbol{A} / \theta=\boldsymbol{A} / \operatorname{ker} g \cong g[\boldsymbol{A}]$, so $\boldsymbol{B} \in \mathrm{Q} .5$

The following quasi-equations prevent us from extending Theorem 12.9 to NRSA, to $V_{\perp}$, and to $V_{\mathbf{f}}$, respectively, as they are not preserved by homomorphic images in these varieties:

$$
\begin{aligned}
\diamond x & =\mathbf{t} \Longrightarrow x=\mathbf{t}, \\
\diamond \perp & =\mathbf{t} \Longrightarrow x=y, \\
\mathbf{f} & =\mathbf{t} \Longrightarrow x=y .
\end{aligned}
$$

\section{Applications}

We can now present some new results about non-integral varieties and logics, which follow from the category equivalences proved here.

Theorem 13.1. Let $\mathrm{K}$ be any variety of (possibly bounded) generalized Sugihara monoids or Sugihara monoids. Then $\mathrm{K}$ has the weak ES property.

Proof. This follows from Corollary 12.5. Theorems 8.7 and 10.5, their bounded extensions (at the conclusion of Section 10) and Theorem 9.2 .

From Theorems 9.1 and 13.1, we infer:

Corollary 13.2. If a variety of (possibly bounded) generalized Sugihara monoids or Sugihara monoids has the amalgamation property, then it has the strong amalgamation property.

Theorem 13.3. The varieties CGSM, PSM and SM have the strong amalgamation property, and therefore the strong ES property. The same applies to their bounded expansions.

Proof. Amalgamation follows from Lemma 12.8, the category equivalences in Theorems 10.3 10.5 and the bounded extensions of these. Then strong amalgamation follows from Corollary 13.2 and the strong ES property from Theorem 9.1 .

On the other hand, most of the varieties mentioned in Theorem 13.1 are not amalgamable; see [21,26, 43].

Theorem 13.4. Every quasivariety of centred generalized Sugihara monoids is a variety.

Proof. This follows from Theorems $9.2,10.3$ and 12.9 .

Theorem 13.4 generalizes the main result of 49, which showed directly that every subquasivariety of PSM is a variety. The present proof is much simpler, modulo the category equivalence.

\footnotetext{
${ }^{5} g$ is the algebraic analogue of 'Prucnal's substitution' from 51. In general, a locally finite variety $\mathrm{K}$ has no subquasivarieties other than varieties iff every finite subdirectly irreducible member of $\mathrm{K}$ embeds into each of its homomorphic pre-images in $\mathrm{K}$; see Gorbunov [28.
} 


\section{Applications to Deductive Systems.}

A formal system $\mathbf{F}$ is said to axiomatize a deductive system $\vdash$ if $\vdash$ and $\vdash_{\mathbf{F}}$ coincide. An extension of $\vdash$ is a deductive system in the same language that is a superset of $\vdash$. It is an axiomatic extension if it has the form $\vdash_{\mathbf{F}^{\prime}}$, where $\mathbf{F}^{\prime}$ adds only axioms (not inference rules) to some axiomatization of $\vdash$.

A postulate $r_{1}, \ldots, r_{n} \vdash s$ is called an admissible rule of $\mathbf{F}$ if its addition to $\mathbf{F}$ yields no new theorems. We say that $\mathbf{F}$ (or $\vdash_{\mathbf{F}}$ ) is structurally complete if its admissible rules are already derivable, i.e., they belong to $\vdash_{\mathbf{F}}$. Hereditary structural completeness makes this demand for the axiomatic extensions of the system as well, and it is equivalent to the claim that every extension is an axiomatic extension (see [50]).

Remark 13.5. For any variety $\mathrm{K}$ of $\mathrm{CRL}$-expansions, the map $\mathrm{M} \mapsto \vdash_{\mathrm{M}}$ defines a lattice anti-isomorphism from the subquasivarieties of $\mathrm{K}$ onto the extensions of $\vdash_{\mathrm{K}}$, and it takes the subvarieties of $\mathrm{K}$ onto the axiomatic extensions of $\vdash_{\mathrm{K}}$. (This follows from results in [11; it is explained in greater generality in [53.)

Remark 13.5 and Theorem 13.4 yield the next result.

\section{Theorem 13.6.}

(i) $\vdash_{\mathrm{CGSM}}$ is hereditarily structurally complete. In particular:

(ii) (49]) The negation-less fragment of $\mathbf{R M}^{\mathbf{t}}$ is hereditarily structurally complete.6

As we pointed out in [26], hereditary structural completeness for IUML follows similarly from the category equivalence between $\mathrm{GA}$ and $\mathrm{OSM}_{\perp}$, because every quasivariety of Gödel algebras is a variety [20].

We say that a formal system $\mathbf{F}$ has the finite Beth property for deduction if $\vdash_{\mathbf{F}}$ has the finite Beth property according to Definitions [11.5] and 11.7. Similarly for the other two Beth properties. This terminology becomes necessary in substructural logics (e.g., $\mathbf{R} \mathbf{M}^{\mathbf{t}}$ ), as these have an implication connective $\rightarrow$ for which the classical deduction theorem may fail. In such cases, there are rival notions of definability, where $\rightarrow$ takes over the role of $\vdash_{\mathbf{F}}$ in Definition 11.5, and we want to avoid confusion.

Theorem 13.7. All axiomatic extensions of $\mathbf{R M}^{\mathbf{t}}$ and all extensions of the negation-less fragment of $\mathbf{R M}^{\mathbf{t}}$ have the finite Beth property for deduction.

Proof. This follows from Remark 13.5, together with Theorems 13.1, 11.2 (iii) and 13.6(ii).

Theorem 13.8. $\mathbf{R M}^{\mathbf{t}}$ and its negation-less fragment have the projective Beth property for deduction.

Proof. This follows from Theorems 11.2(iv) and 13.3

Note that relevant and many-valued logics rarely possess even the finite Beth property [9, 47, 59]. It seems to be difficult to prove Theorems 13.1 and 13.3 and Corollary 13.2 (and therefore Theorems 13.7 and 13.8) without using the category equivalences revealed here.

For the reasons given before Theorem 13.7, a formal system $\mathbf{F}$ is said to have the deductive interpolation property if $\vdash_{\mathbf{F}}$ has the interpolation property in the

\footnotetext{
${ }^{6} \mathbf{R M}^{\mathbf{t}}$ itself is not structurally complete, e.g., $x \leftrightarrow \neg x \vdash y$ is admissible but not derivable.
} 
sense of Definition 11.3(i). In substructural logics, the rival (implicative) form of interpolation is usually called Craig interpolation.

It follows from Theorems $11.2(\mathrm{v})$ and 13.3 and the congruence extensibility of [involutive] CRLs that

$\mathbf{R M}^{\mathbf{t}}$ and its negation-less fragment have the deductive interpola-

tion property,

but the former assertion is not new. Indeed, R. K. Meyer established Craig interpolation for $\mathbf{R M}^{\mathbf{t}}$ in [46], inferring deductive interpolation as a corollary (see [3] also). Recently, E. Marchioni and G. Metcalfe have shown that deductive and Craig interpolation coincide for axiomatic extensions of $\mathbf{R} \mathbf{M}^{\mathbf{t}}$, and they have isolated the axiomatic extensions with this property by determining the amalgamable varieties of Sugihara monoids [43].

\section{Genesis OF THE FUnCTOR $S$}

To conclude this paper, we explain how we arrived at the definition of $\boldsymbol{S}(\boldsymbol{A})$ in Section 8

An algebraic characterization of categorical equivalence for arbitrary pairs of quasivarieties is provided in R. McKenzie's paper 44. This makes it easier, in principle, to establish an equivalence without producing two explicit functors. The characterization involves two constructions: idempotent images and matrix powers. We recall the definitions here.

Given an algebra $\boldsymbol{A}$ and a positive integer $k$, let $T_{k}(\boldsymbol{A})$ be the set of all $k$-ary terms in the language of $\boldsymbol{A}$, and let $T(\boldsymbol{A})=\bigcup_{0<n \in \omega} T_{n}(\boldsymbol{A})$. For a unary term $\sigma$ of $\boldsymbol{A}$, the $\sigma$-image of $\boldsymbol{A}$ is the algebra

$$
\boldsymbol{A}(\sigma)=\left\langle\sigma[A] ;\left\{t_{\sigma}: t \in T(\boldsymbol{A})\right\}\right\rangle,
$$

where, for each positive $n$ and each $t \in T_{n}(\boldsymbol{A})$,

$$
t_{\sigma}^{\boldsymbol{A}(\sigma)}\left(a_{1}, \ldots, a_{n}\right)=\sigma^{\boldsymbol{A}}\left(t^{\boldsymbol{A}}\left(a_{1}, \ldots, a_{n}\right)\right) \text { for } a_{1}, \ldots, a_{n} \in \sigma[A] .
$$

Thus, every term of $\boldsymbol{A}$ gives rise to a basic operation of $\boldsymbol{A}(\sigma)$.

For each positive $n$, the $n$-th matrix power of $\boldsymbol{A}$ is the algebra

$$
\boldsymbol{A}^{[n]}=\left\langle A^{n} ;\left\{m_{t}: t \in\left(T_{k n}(\boldsymbol{A})\right)^{n} \text { for some positive } k \in \omega\right\}\right\rangle,
$$

where, for each $t=\left\langle t_{1}, \ldots, t_{n}\right\rangle \in\left(T_{k n}(\boldsymbol{A})\right)^{n}$, we define $m_{t}:\left(A^{n}\right)^{k} \longrightarrow A^{n}$ as follows: if $a_{j}=\left\langle a_{j 1}, \ldots, a_{j n}\right\rangle \in A^{n}$ for $j=1, \ldots, k$, then

$$
\pi_{i}\left(m_{t}\left(a_{1}, \ldots, a_{k}\right)\right)=t_{i}^{\boldsymbol{A}}\left(a_{11}, \ldots, a_{1 n}, \ldots, a_{k 1}, \ldots, a_{k n}\right)
$$

for each of the $n$ projections $\pi_{i}: A^{n} \longrightarrow A$. In short, $\boldsymbol{A}^{[n]}$ has $A^{n}$ as its universe, and its basic operations are all conceivable operations on $n$-tuples that can be defined using the terms of $\boldsymbol{A}$.

Let $\mathrm{K}$ be a class of similar algebras. A unary term $\sigma$ of $\mathrm{K}$ is said to be idempotent in $\mathrm{K}$ if $\mathrm{K}$ satisfies $\sigma(\sigma(x))=\sigma(x)$, and invertible in $\mathrm{K}$ if $\mathrm{K}$ satisfies $x=t\left(\sigma\left(t_{1}(x)\right), \ldots, \sigma\left(t_{r}(x)\right)\right)$ for some positive integer $r$, some unary terms $t_{1}, \ldots, t_{r}$ and some $r$-ary term $t$. Let $\mathrm{K}(\sigma)$ and $\mathrm{K}^{[n]}$ denote the isomorphic closures of $\{\boldsymbol{A}(\sigma): \boldsymbol{A} \in \mathrm{K}\}$ and $\left\{\boldsymbol{A}^{[n]}: \boldsymbol{A} \in \mathrm{K}\right\}$, respectively. Thus, $\mathrm{K}^{[1]}(\sigma)=\mathrm{K}(\sigma)$. If K is a [quasi]variety then so are $\mathrm{K}(\sigma)$ and $\mathrm{K}^{[n]}$, provided that $\sigma$ is idempotent in $\mathrm{K}$. McKenzie's result, restricted to quasivarieties, is as follows. 
Theorem 14.1 (McKenzie 44]). Two quasivarieties $\mathrm{K}$ and $\mathrm{M}$ are categorically equivalent iff there is a positive integer $n$ and an invertible idempotent term $\sigma$ of $\mathrm{K}^{[n]}$ such that $\mathrm{M}$ is termwise equivalent to $\mathrm{K}^{[n]}(\sigma)$.

In 26], we proved directly that $\operatorname{OSM}(\sigma)$ and RSA are termwise equivalent, where $\sigma(x)$ is $x \wedge \mathbf{t}$. This $\sigma$ is obviously still idempotent in GSM. It is also invertible there, because Lemma 3.4 says that GSM satisfies

$$
x=(x \wedge \mathbf{t}) \cdot\left(x^{*} \wedge \mathbf{t}\right)^{*}=t\left(\sigma\left(t_{1}(x)\right), \sigma\left(t_{2}(x)\right)\right),
$$

where

$$
t_{1}(x) \text { is } x \text { and } t_{2}(x) \text { is } x^{*} \text { and } t(x, y) \text { is } x \cdot y^{*} .
$$

If $\boldsymbol{A} \in \mathrm{GSM}$, then $\boldsymbol{A}_{\diamond}^{-}$is a reduct of $\boldsymbol{A}(\sigma)$ (see Example 7.3), and it seems reasonable to hope that NRSA is termwise equivalent to $\operatorname{GSM}(\sigma)$. This assertion amounts to the following:

for every term $s$ of GSM, there exists a term $r$ of NRSA such that, for every $\boldsymbol{A} \in \mathrm{GSM}$, we have $\left.(s \wedge \mathbf{t})^{\boldsymbol{A}}\right|_{A^{-}}=r^{\boldsymbol{A}_{\diamond}^{-}}$.

Unfortunately, the terms of GSM are not as tame as those of OSM. We shall return to the above claim shortly. For the moment, we make do with two special cases, set out in the next lemma. Note that

$$
x \rightarrow_{\sigma} y \text { abbreviates }(x \rightarrow y) \wedge \mathbf{t}
$$

in the language of GSM.

Lemma 14.2. Let $\boldsymbol{A}$ be a generalized Sugihara monoid, with $a, b \in A^{-}$. Then

$$
\begin{aligned}
& a \cdot b^{*}=\left(a \rightarrow_{\sigma} b^{* *}\right) \rightarrow\left(\left(a \rightarrow_{\sigma} b^{* *}\right) \rightarrow_{\sigma} a\right), \\
& \left(a \cdot b^{*}\right)^{*}=a \rightarrow b^{* *} .
\end{aligned}
$$

Consequently, by the contraction property,

$$
\begin{aligned}
& \left(a \cdot b^{*}\right) \wedge \mathbf{t}=\left(a \rightarrow{ }^{\boldsymbol{A}_{\diamond}^{-}} \diamond b\right) \rightarrow^{\boldsymbol{A}_{\diamond}^{-}} a, \\
& \left(a \cdot b^{*}\right)^{*} \wedge \mathbf{t}=a \rightarrow^{\boldsymbol{A}_{\diamond}^{-}} \diamond b .
\end{aligned}
$$

Proof. Since CRLs satisfy $(x \cdot y) \rightarrow z=x \rightarrow(y \rightarrow z)$, they also satisfy (45). So, it suffices to prove (44), and we may assume without loss of generality that $\boldsymbol{A}$ is totally ordered. The proof is accomplished with the aid of Theorem 3.2, by considering cases.

Taking the term equivalence of $\operatorname{GSM}(\sigma)$ and NRSA as a working hypothesis, and using the analysis of [44, we can partially predict the form of a category equivalence functor $S$ from NRSA to GSM. More exactly, we can determine analytically the universe of the GSM-image of each $\boldsymbol{A} \in$ NRSA. Indeed, by the symmetry of categorical equivalence and Theorem 14.1. our hypothesis implies that GSM is termwise equivalent to $\operatorname{NRSA}^{[m]}(\tau)$ for some positive integer $m$ and some invertible idempotent term $\tau$ of $\operatorname{NRSA}^{[m]}$. Because the term $t$ in (43) is binary, we can predict from [44, Remark 2] that $m=2$, so $\tau$ has the form $\left\langle\tau_{1}(x, y), \tau_{2}(x, y)\right\rangle$ for some binary terms $\tau_{1}$ and $\tau_{2}$ of NRSA.

Since NRSA and $\operatorname{GSM}(\sigma)$ were assumed to be termwise equivalent, GSM will be termwise equivalent to $\operatorname{GSM}(\sigma)^{[2]}\left(\tau^{\prime}\right)$ for a suitable invertible idempotent term $\tau^{\prime}$ of 
$\operatorname{GSM}(\sigma)^{[2]}$. As it happens, we can solve for $\tau^{\prime}$, and hence for $\tau$. Indeed, Remark 2 of [44] tells us that

$$
\tau^{\prime}(x, y)=\left\langle\sigma\left(t_{1}(t(x, y))\right), \sigma\left(t_{2}(t(x, y))\right)\right\rangle=\left\langle\left(x \cdot y^{*}\right) \wedge \mathbf{t},\left(x \cdot y^{*}\right)^{*} \wedge \mathbf{t}\right\rangle
$$

will be a solution and, using (46) and (47), we obtain

$$
\tau(x, y)=\langle(x \rightarrow \diamond y) \rightarrow x, x \rightarrow \diamond y\rangle .
$$

Now let $\boldsymbol{A}$ be a nuclear relative Stone algebra. According to [44], the equivalence functor $S$ can be chosen so that the GSM-image $\boldsymbol{S}(\boldsymbol{A})$ of $\boldsymbol{A}$ is termwise equivalent to $\boldsymbol{A}^{[2]}(\tau)$. Its universe $S(\boldsymbol{A})$ must then consist of the fixed points of $\tau$ in $A \times A$, because $\tau$ is idempotent. In other words,

$$
S(\boldsymbol{A})=\left\{\left\langle a, a^{\prime}\right\rangle \in A \times A:\left(a \rightarrow \diamond a^{\prime}\right) \rightarrow a=a \text { and } a \rightarrow \diamond a^{\prime}=a^{\prime}\right\} .
$$

It follows readily that

$$
S(\boldsymbol{A})=\left\{\left\langle a, a^{\prime}\right\rangle \in A \times A: a \vee a^{\prime}=\mathbf{t} \text { and } \diamond a^{\prime}=a^{\prime}\right\}
$$

(use (14), (15) and Lemma 4.1).

The general theory in 44 deals with the full clone of term operations of a class, so it doesn't tell us how to isolate appropriate basic operations for $\boldsymbol{S}(\boldsymbol{A})$. There seems to be no analytical way to determine these in advance. In [26], where we dealt only with the special case of RSA and OSM, our simpler definitions of $\boldsymbol{\cdot} \boldsymbol{S}(\boldsymbol{A})$ and $\rightarrow \boldsymbol{S}(\boldsymbol{A})$ were partly inspired by Dunn's semantic analysis of the logic $\mathbf{R}-$ mingle (see $[19]$ ), but it is not obvious how to incorporate $\diamond$ when constructing the operations of generalized Sugihara monoids.

In [26, Sec. 9], however, we noted a serendipitous connection between our construction and an earlier one of P. H. Chu (discussed in [7, 13, 56, 58]). When applied to any integral non-trivial CRL $\boldsymbol{A}$, Chu's construction yields a non-integral involutive CRL with universe $A \times A$, but it fails to preserve the idempotence of $\boldsymbol{A}$, so it is not directly applicable to our investigation. Nevertheless, when $\boldsymbol{A} \in$ NRSA, it can be shown that Chu's operations $\wedge, \vee$ and $\mathbf{t}$ are invariant under the function $\tau$ in (48), making them plausible candidates for $\wedge^{S(A)}, \vee^{S(A)}$ and $\mathbf{t}^{\boldsymbol{S}(\boldsymbol{A})}$. (They also feature in an earlier and simpler construction of J. A. Kalman [35], which does not deal with operations like $\cdot$ and $\rightarrow$.) Chu's $\cdot$ and $\rightarrow$ are not $\tau$-invariant, but their $\tau$-images become experimental candidates for. $\boldsymbol{S}(\boldsymbol{A})$ and $\rightarrow \boldsymbol{S}(\boldsymbol{A})$, because $\tau$ is idempotent. These images are very complicated in the first instance, but they can be shown to coincide, over $S(\boldsymbol{A})$, with the ones in Section 8 , provided that $\boldsymbol{A} \in$ NRSA. In the special case where $\boldsymbol{A} \in$ RSA, i.e., $\nabla^{\boldsymbol{A}}$ is the identity function on $A$, our $\neg \boldsymbol{S}(\boldsymbol{A})$ reduces to Chu's (and Kalman's) $\neg$, which is then $\tau$-invariant. It ceases to be $\tau$-invariant when $\boldsymbol{A}$ merely belongs to $\mathrm{X}$, i.e., when the intended Sugihara monoid $\boldsymbol{S}(\boldsymbol{A})$ is not expected to be odd. But in that case, our inferential definition of $\neg \boldsymbol{S}(\boldsymbol{A})$ in (38) is a natural idea.

These considerations motivate the operations of $\boldsymbol{S}(\boldsymbol{A})$, but they contain no guarantee of success, and no short-cut to the work done in Section 8 . Since the nuclear negative cone functor turns out to reverse $S$, our working hypothesis can be confirmed via the next result - which is the case $n=1$ of [44, Thm.6.1].

Theorem 14.3 (McKenzie). Let $F: \mathrm{K} \longrightarrow \mathrm{M}$ be a category equivalence between quasivarieties, and let $\sigma$ be an invertible idempotent unary term of $\mathrm{K}$. Let $\boldsymbol{A}$ be the free 1-generated algebra in $\mathrm{M}$, and $\boldsymbol{B}$ the algebra in $\mathrm{K}$ freely generated by a single generator $b$ subject to the relation $\sigma(b)=b$. 
If $F(\boldsymbol{B}) \cong \boldsymbol{A}$, then $\mathrm{K}(\sigma)$ and $\mathrm{M}$ are termwise equivalent, and the algebras $\boldsymbol{C}(\sigma)$ and $F(\boldsymbol{C})$ are termwise equivalent for all $\boldsymbol{C} \in \mathrm{K}$.

Corollary 14.4. The varieties $\operatorname{GSM}(\sigma)$ and NRSA are termwise equivalent, where $\sigma(x):=x \wedge \mathbf{t}$.

Proof. Set $\mathrm{K}=\mathrm{GSM}$ and $\mathrm{M}=$ NRSA. Let $F$ be the nuclear negative cone functor, $\boldsymbol{A}$ the algebra in Example 8.4 with $\diamond d=c$, and $\boldsymbol{B}=\boldsymbol{S}(\boldsymbol{A})$. Now apply Theorems 8.7 and 14.3 .

Although we need a description of $S$ in Section 10, a direct proof of Corollary 14.4 would yield categorical equivalence for GSM and NRSA, without recourse to $S$. And it would be enough to show that for any term $s$ involving only $\cdot, \rightarrow$ and ${ }^{*}$, there exists a term $r$ of NRSA such that $\left.(s \wedge \mathbf{t})^{\boldsymbol{A}}\right|_{A^{-}}=r^{\boldsymbol{A}_{\diamond}^{-}}$for every generalized Sugihara monoid $\boldsymbol{A}$ (cf. the proof of [26, Thm.5.5]). This is made plausible by Lemma 14.2 and the next proposition, the non-trivial parts of which can be verified using Theorem 3.2 .

Proposition 14.5. Let $a, b, c$ be negative elements of a generalized Sugihara monoid A. Then

(i) $a \cdot b=a \wedge b$,

(ii) $a^{*} \cdot b^{*}=(a \wedge b)^{*}$,

(iii) $a^{*} \rightarrow b=a^{* *} \wedge b$,

(iv) $a \rightarrow b^{*}=(a \wedge b)^{*}$,

(v) $c \rightarrow(a \rightarrow b)=(c \wedge a) \rightarrow b$,

(vi) $\left(a \cdot b^{*}\right) \rightarrow c=a \rightarrow\left(b^{* *} \wedge c\right)$,

(vii) $a \rightarrow b=\left(a \rightarrow_{\sigma} b\right) \cdot\left[\left(a \rightarrow_{\sigma} b\right) \rightarrow_{\sigma} a\right]^{*}$.

With the exception of (vii), the right hand sides of these equations contain no occurrence of $\cdot$, and the $\cdot$ in (vii) can be eliminated by a subsequent application of (44). Thus, when $a, b, c \in A^{-}$and $s^{\boldsymbol{A}}(a, b, c)$ is the right hand side of one of the above equations, we can easily find an NRSA-term $r$ such that

$$
s^{\boldsymbol{A}}(a, b, c) \wedge \mathbf{t}=r^{\boldsymbol{A}_{\diamond}^{-}}(a, b, c) .
$$

It seems likely that, by composing these seven re-write rules and (44)-(47) judiciously, we can extract a suitable $r$ from every GSM-term $s$. In the final step, we would trade in all occurrences of the GSM-symbols $\rightarrow_{\sigma}$ and ${ }^{* *}$ for the NRSAsymbols $\rightarrow$ and $\diamond$, respectively. In the penultimate step, where ' $\wedge \mathbf{t}$ ' is applied, the contraction property allows us to shorten any $\left(a \rightarrow\left(a \rightarrow_{\sigma} b\right)\right) \wedge \mathbf{t}$ to $a \rightarrow_{\sigma} b$. The following examples are obtained in this way.

$$
\begin{array}{cc}
s & r \\
(x \rightarrow y)^{*} & (x \rightarrow y) \rightarrow \diamond((x \rightarrow y) \rightarrow x) \\
(x \rightarrow y) \rightarrow z & (x \rightarrow y) \rightarrow[z \wedge \diamond((x \rightarrow y) \rightarrow x)] \\
x \rightarrow\left(y \cdot z^{*}\right) & (x \wedge(y \rightarrow \diamond z)) \rightarrow((y \rightarrow \diamond z) \rightarrow y) \\
(x \rightarrow y) \cdot z & w \rightarrow(z \wedge(x \rightarrow y))
\end{array}
$$

In the last line, $w$ abbreviates $(z \wedge(x \rightarrow y)) \rightarrow \diamond((x \rightarrow y) \rightarrow x)$.

These calculations pre-date the proof of Theorem 8.7 but they gave us enough confidence in Corollary 14.4 to develop the material in Section 8 . 


\section{REFERENCES}

[1] Paolo Agliano, Ternary deduction terms in residuated structures, Acta Sci. Math. (Szeged) 64 (1998), no. 3-4, 397-429. MR1666002 (2000a:08005)

[2] Alan Ross Anderson and Nuel D. Belnap Jr., Entailment, Princeton University Press, Princeton, N. J., 1975. Volume I: The logic of relevance and necessity; With contributions by J. Michael Dunn and Robert K. Meyer, and further contributions by John R. Chidgey, J. Alberto Coffa, Dorothy L. Grover, Bas van Fraassen, Hugues LeBlanc, Storrs McCall, Zane Parks, Garrel Pottinger, Richard Routley, Alasdair Urquhart and Robert G. Wolf. MR.0406756 (53 \#10542)

[3] Arnon Avron, On an implication connective of RM, Notre Dame J. Formal Logic 27 (1986), no. 2, 201-209, DOI 10.1305/ndjfl/1093636612. MR842148(88c:03019)

[4] Arnon Avron, The semantics and proof theory of linear logic, Theoret. Comput. Sci. 57 (1988), no. 2-3, 161-184, DOI 10.1016/0304-3975(88)90037-0. MR.960102 (90k:68100)

[5] B. Banaschewski, On categories of algebras equivalent to a variety, Algebra Universalis 16 (1983), no. 2, 264-267, DOI 10.1007/BF01191779. MR692271 (84g:08025)

[6] Paul Bankston and Ralph Fox, On categories of algebras equivalent to a quasivariety, Algebra Universalis 16 (1983), no. 2, 153-158, DOI 10.1007/BF01191762. MR692254 (84g:08026)

[7] Michael Barr, *-autonomous categories, Lecture Notes in Mathematics, vol. 752, Springer, Berlin, 1979. With an appendix by Po Hsiang Chu. MR.550878 (81h:18008)

[8] J. Barwise and S. Feferman (eds.), Model-theoretic logics, Perspectives in Mathematical Logic, Springer-Verlag, New York, 1985. Edited by J. Barwise and S. Feferman. MR819531 (87g:03033)

[9] W. J. Blok and Eva Hoogland, The Beth property in algebraic logic, Studia Logica 83 (2006), no. 1-3, 49-90, DOI 10.1007/s11225-006-8298-0. MR2250105 (2007e:03111)

[10] Wim J. Blok and Don L. Pigozzi, Local deduction theorems in algebraic logic, Algebraic logic (Budapest, 1988), Colloq. Math. Soc. János Bolyai, vol. 54, North-Holland, Amsterdam, 1991, pp. 75-109. MR.1153419 (93g:03053)

[11] W. J. Blok and Don Pigozzi, Algebraizable logics, Mem. Amer. Math. Soc. 77 (1989), no. 396, vi+78. MR973361 (90d:03140)

[12] Stanley Burris and H. P. Sankappanavar, A course in universal algebra, Graduate Texts in Mathematics, vol. 78, Springer-Verlag, New York, 1981. MR648287(83k:08001)

[13] Manuela Busaniche and Roberto Cignoli, Residuated lattices as an algebraic semantics for paraconsistent Nelson's logic, J. Logic Comput. 19 (2009), no. 6, 1019-1029, DOI 10.1093/logcom/exp028. MR2565914(2011a:03025)

[14] Janusz Czelakowski, Local deductions theorems, Studia Logica 45 (1986), no. 4, 377-391, DOI 10.1007/BF00370271. MR884146 (88g:03045)

[15] Janusz Czelakowski, Protoalgebraic logics, Trends in Logic-Studia Logica Library, vol. 10, Kluwer Academic Publishers, Dordrecht, 2001. MR1828895 (2002f:03001)

[16] Janusz Czelakowski and Wiesław Dziobiak, Congruence distributive quasivarieties whose finitely subdirectly irreducible members form a universal class, Algebra Universalis 27 (1990), no. 1, 128-149, DOI 10.1007/BF01190258. MR1025840 (91i:08013)

[17] Janusz Czelakowski and Don Pigozzi, Amalgamation and interpolation in abstract algebraic logic, Models, algebras, and proofs (Bogotá, 1995), Lecture Notes in Pure and Appl. Math., vol. 203, Dekker, New York, 1999, pp. 187-265. MR1686920 (2000e:03175)

[18] J. Michael Dunn, Algebraic completeness results for $R$-mingle and its extensions, J. Symbolic Logic 35 (1970), 1-13. MR0288008 (44 \#5206)

[19] J. Michael Dunn, A Kripke-style semantics for $R$-mingle using a binary accessibility relation, Studia Logica 35 (1976), no. 2, 163-172. MR0441691(56 \#90)

[20] Wojciech Dzik and Andrzej Wroński, Structural completeness of Gödel's and Dummett's propositional calculi (English, with Polish and Russian summaries), Studia Logica 32 (1973), 69-75. MR 0347579 (50 \#82)

[21] D. M. Gabbay and L. Maksimova, Interpolation and definability, Oxford Logic Guides, vol. 46, The Clarendon Press Oxford University Press, Oxford, 2005. Modal and intuitionistic logics. MR2153890(2006d:03002)

[22] Nikolaos Galatos, Varieties of residuated lattices, ProQuest LLC, Ann Arbor, MI, 2003. Thesis (Ph.D.)-Vanderbilt University. MR2704503 
[23] Nikolaos Galatos, Peter Jipsen, Tomasz Kowalski, and Hiroakira Ono, Residuated lattices: an algebraic glimpse at substructural logics, Studies in Logic and the Foundations of Mathematics, vol. 151, Elsevier B. V., Amsterdam, 2007. MR2531579 (2011d:03033)

[24] Nikolaos Galatos, Jeffrey S. Olson, and James G. Raftery, Irreducible residuated semilattices and finitely based varieties, Rep. Math. Logic 43 (2008), 85-108. MR2417724 (2009j:03048)

[25] Nikolaos Galatos and Hiroakira Ono, Algebraization, parametrized local deduction theorem and interpolation for substructural logics over FL, Studia Logica 83 (2006), no. 1-3, 279-308, DOI 10.1007/s11225-006-8305-5. MR2250112 (2007d:03035)

[26] N. Galatos and J. G. Raftery, A category equivalence for odd Sugihara monoids and its applications, J. Pure Appl. Algebra 216 (2012), no. 10, 2177-2192, DOI 10.1016/j.jpaa.2012.02.006. MR2925812

[27] Jean-Yves Girard, Linear logic, Theoret. Comput. Sci. 50 (1987), no. 1, 101, DOI 10.1016/0304-3975(87)90045-4. MR899269 (89m:03057)

[28] V. A. Gorbunov, Lattices of quasivarieties (Russian), Algebra i Logika 15 (1976), no. 4, 436-457, 487-488. MR0447076 (56 \#5391)

[29] James B. Hart, Lori Rafter, and Constantine Tsinakis, The structure of commutative residuated lattices, Internat. J. Algebra Comput. 12 (2002), no. 4, 509-524, DOI 10.1142/S0218196702001048. MR:1919685 (2003g:06008)

[30] Leon Henkin, J. Donald Monk, and Alfred Tarski, Cylindric algebras. Part II, Studies in Logic and the Foundations of Mathematics, vol. 115, North-Holland Publishing Co., Amsterdam, 1985. MR781930 (86m:03095b)

[31] Eva Hoogland, Algebraic characterizations of various Beth definability properties, Studia Logica 65 (2000), no. 1, 91-112, DOI 10.1023/A:1005295109904. Abstract algebraic logic, I (Barcelona, 1997). MR1781785 (2001j:03063)

[32] E. Hoogland, Definability and interpolation: model-theoretic investigations, Ph.D. Thesis, Institute for Logic, Language and Computation, University of Amsterdam, 2001.

[33] John R. Isbell, Epimorphisms and dominions, Proc. Conf. Categorical Algebra (La Jolla, Calif., 1965), Springer, New York, 1966, pp. 232-246. MR0209202 (35 \#105a)

[34] Bjarni Jónsson, Algebras whose congruence lattices are distributive, Math. Scand. 21 (1967), 110-121 (1968). MR0237402(38 \#5689)

[35] J. A. Kalman, Lattices with involution, Trans. Amer. Math. Soc. 87 (1958), 485-491. MR0095135 (20 \#1641)

[36] Hitoshi Kihara and Hiroakira Ono, Interpolation properties, Beth definability properties and amalgamation properties for substructural logics, J. Logic Comput. 20 (2010), no. 4, 823-875, DOI 10.1093/logcom/exn084. MR2670238(2011k:03049)

[37] E. W. Kiss, L. Márki, P. Pröhle, and W. Tholen, Categorical algebraic properties. A compendium on amalgamation, congruence extension, epimorphisms, residual smallness, and injectivity, Studia Sci. Math. Hungar. 18 (1982), no. 1, 79-140. MR759319 (85k:18003)

[38] G. Kreisel, Explicit definability in intuitionistic logic, J. Symbolic Logic 25 (1960), 389-390.

[39] J. Łoś and R. Suszko, Remarks on sentential logics, Nederl. Akad. Wetensch. Proc. Ser. A 61 = Indag. Math. 20 (1958), 177-183. MR0098670 (20 \#5125)

[40] L.L. Maksimova, Craig's interpolation theorem and amalgamated varieties of pseudoboolean algebras, Algebra and Logic 16 (1977), 643-681.

[41] L.L. Maksimova, Craig's interpolation theorem and amalgamable manifolds, Soviet Math. Doklady 18 (1977), 1511-1514.

[42] E. Marchioni, G. Metcalfe, Interpolation properties for uninorm based logics, Proceedings of ISMVL 2010, IEEE Computer Society Press (2010), 205-210.

[43] E. Marchioni and G. Metcalfe, Craig interpolation for semilinear substructural logics, MLQ Math. Log. Q. 58 (2012), no. 6, 468-481, DOI 10.1002/malq.201200004. MR2997034

[44] Ralph McKenzie, An algebraic version of categorical equivalence for varieties and more general algebraic categories, Logic and algebra (Pontignano, 1994), Lecture Notes in Pure and Appl. Math., vol. 180, Dekker, New York, 1996, pp. 211-243. MR.1404941 (98a:08010)

[45] George Metcalfe and Franco Montagna, Substructural fuzzy logics, J. Symbolic Logic 72 (2007), no. 3, 834-864, DOI 10.2178/jsl/1191333844. MR2354903 (2008k:03057)

[46] R.K. Meyer, Relevantly interpolating in RM, Technical Report 9, Logic Group RSSS, Australian National University, 1980. 
[47] Franco Montagna, Interpolation and Beth's property in propositional many-valued logics: a semantic investigation, Ann. Pure Appl. Logic 141 (2006), no. 1-2, 148-179, DOI 10.1016/j.apal.2005.11.001. MR2229934 (2007c:03034)

[48] Jeffrey S. Olson, Subdirectly irreducible residuated semilattices and positive universal classes, Studia Logica 83 (2006), no. 1-3, 393-406, DOI 10.1007/s11225-006-8310-8. MR2250117 (2007g:06002)

[49] Jeffrey S. Olson and James G. Raftery, Positive Sugihara monoids, Algebra Universalis 57 (2007), no. 1, 75-99, DOI 10.1007/s00012-007-2022-4. MR2326927(2008g:03041)

[50] J. S. Olson, J. G. Raftery, and C. J. van Alten, Structural completeness in substructural logics, Log. J. IGPL 16 (2008), no. 5, 455-495, DOI 10.1093/jigpal/jzn014. MR2453364 (2009h:03030)

[51] Tadeusz Prucnal, On the structural completeness of some pure implicational propositional calculi (English, with Polish and Russian summaries), Studia Logica 30 (1972), 45-52. MR.0317878 (47 \#6427)

[52] Tadeusz Prucnal and Andrzej Wroński, An algebraic characterization of the notion of structural completeness, Polish Acad. Sci. Inst. Philos. Sociol. Bull. Sect. Logic 3 (1974), no. 1, 30-33. MR0403950(53 \#7759)

[53] J. G. Raftery, Correspondences between Gentzen and Hilbert systems, J. Symbolic Logic 71 (2006), no. 3, 903-957, DOI 10.2178/jsl/1154698583. MR2251547 (2007e:03100)

[54] J. G. Raftery, Representable idempotent commutative residuated lattices, Trans. Amer. Math. Soc. 359 (2007), no. 9, 4405-4427, DOI 10.1090/S0002-9947-07-04235-3. MR.2309191 (2009b:03063)

[55] Claus Michael Ringel, The intersection property of amalgamations, J. Pure Appl. Algebra 2 (1972), 341-342. MR0332919 (48 \#11244)

[56] Kimmo I. Rosenthal, Quantales and their applications, Pitman Research Notes in Mathematics Series, vol. 234, Longman Scientific \& Technical, Harlow, 1990. MR 1088258 (92e:06028)

[57] A. S. Troelstra, Lectures on linear logic, CSLI Lecture Notes, vol. 29, Stanford University Center for the Study of Language and Information, Stanford, CA, 1992. MR1163373(93i:03083)

[58] C. Tsinakis and A. M. Wille, Minimal varieties of involutive residuated lattices, Studia Logica 83 (2006), no. 1-3, 407-423, DOI 10.1007/s11225-006-8311-7. MR2250118 (2008a:06005)

[59] Alasdair Urquhart, Beth's definability theorem in relevant logics, Logic at work, Stud. Fuzziness Soft Comput., vol. 24, Physica, Heidelberg, 1999, pp. 229-234. MR1720799|(2000f:03068)

Department of Mathematics, University of Denver, 2360 S. Gaylord Street, Denver, Colorado 80208

E-mail address: ngalatos@du.edu

Department of Mathematics and Applied Mathematics, University of Pretoria, Private Bag X20, Hatfield, Pretoria 0028, South Africa

E-mail address: james.raftery@up.ac.za 